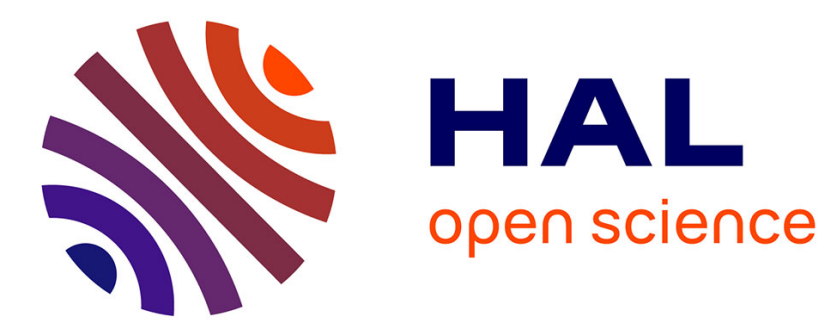

\title{
Les contraintes de la création et de la reprise d'entreprise: une application aux régions françaises
}

Rafik Abdesselam, Jean Bonnet, Nicolas Le Pape

\section{To cite this version:}

Rafik Abdesselam, Jean Bonnet, Nicolas Le Pape. Les contraintes de la création et de la reprise d'entreprise: une application aux régions françaises. Cahiers d'Economie et de Sociologie Rurales, 2004, nº72, 3ième trimestre, pp.33-68. hal-00149382

\section{HAL Id: hal-00149382 \\ https://hal.science/hal-00149382}

Submitted on 12 Nov 2007

HAL is a multi-disciplinary open access archive for the deposit and dissemination of scientific research documents, whether they are published or not. The documents may come from teaching and research institutions in France or abroad, or from public or private research centers.
L'archive ouverte pluridisciplinaire HAL, est destinée au dépôt et à la diffusion de documents scientifiques de niveau recherche, publiés ou non, émanant des établissements d'enseignement et de recherche français ou étrangers, des laboratoires publics ou privés. 


\title{
Les contraintes de la création et de la reprise d'entreprises : une application aux régions françaises
}

\author{
Rafik Abdesselam*, Jean Bonnet* et Nicolas Le Pape*
}

Juin 2004

Publié dans les cahiers d'Economie et de Sociologie Rurales, $n^{\circ} 72, p p .33-68,2004$.

*GEMMA, Université de Caen, France.

e-mails :abdesselam@econ.unicaen.fr ; bonnet@econ.unicaen.fr ; lepape@econ.unicaen.fr Esplanade de la Paix,

14032 Caen, Cedex, France. 
Abstract :

The decision to start up or to take over an enterprise is induced both by the social and personal situation of the individual and by an environment which is more or less favourable to entrepreneurship. In this environment, we identify several constraints such as financial, technical, informational and entrepreneurial networking constraints. Data Analysis Methods allow us to build models of new entrepreneurs based on the type of constraint they are confronted to and to characterize the evolution of the employment associated with these profiles. At a national level, less constrained individuals who take-over a firm are more prone to create jobs. This is not the case for new entrepreneurs (ex-nihilo). In this paper, we also describe the heterogeneity of French regions. For instance, the West part of France is representative of less constrained entrepreneurs. The profile of constrained new entrepreneurs (ex-nihilo) characterizes Provence-Alpes-Côte d'Azur and Languedoc-Roussillon and is associated with a rather unfavourable evolution of employment. By contrast, other regions (Haute-Normandie and Nord-Pas-de-Calais) exhibit the profile of constrained new entrepreneurs associated with a favourable evolution of employment in these firms. A regional public policy which alleviates the constraints borne by new entrepreneurs with the aim of promoting local employment does not necessarily give positive results.

Key-words: Starting-up/Taking over firms, employment, regions, Data analysis Methods

\section{Résumé :}

La décision de créer ou de reprendre une entreprise est déterminée par la situation sociale et personnelle de l'individu et par un environnement plus ou moins propice à l'événement entrepreneurial. Dans cet environnement un certain nombre de contraintes peuvent être identifiées, contrainte financière, contrainte technique, contrainte d'information et contrainte d'insertion dans les réseaux entrepreneuriaux. Nous caractérisons l'hétérogénéité des régions françaises en construisant des modèles de créateurs et de repreneurs selon la nature des contraintes qu'ils subissent. Ces modèles sont construits à l'aide des méthodes de la statistique exploratoire multidimensionnelle appliquées à la base de données issue de l'enquête SINE (Système d'Informations sur les Nouvelles Entreprises). Les régions de l'Ouest de la France, traditionnellement considérées comme des régions de «culture entrepreneuriale», sont représentatives des modèles de créateurs et de repreneurs les moins contraints. L'hétérogénéité des régions françaises est également appréhendée en considérant la création d'emplois des créateurs et des repreneurs d'entreprises. Les régions qui enregistrent les moins bonnes performances (échec entrepreneurial et baisse de l'emploi) à la fois pour les créateurs et les repreneurs d'entreprises sont Provence-Alpes-Côte-d'Azur et Languedoc-Roussillon ainsi que la région capitale. En revanche cinq régions françaises présentent une évolution très favorable de l'emploi par les créateurs et les repreneurs d'entreprises: Midi-Pyrénées, Rhône-Alpes, Poitou-Charentes, Bourgogne et Champagne-Ardenne. Nous recherchons dans l'espace régional à quels profils correspondent les nouveaux entrepreneurs (créateurs et repreneurs) les plus aptes à promouvoir l'emploi. Ces profils sont construits en fonction des quatre principales contraintes identifiées. En Provence-Alpes-Côte-d'Azur et Languedoc-Roussillon le modèle non contraint domine pour les créateurs mais il est associé à une évolution défavorable de l'emploi dans ces entreprises. En revanche dans d'autres régions françaises (Midi-Pyrénées, Nord Pas de Calais et HauteNormandie...) la présence de contraintes au démarrage est une caractéristique des créateurs sans pour autant être un handicap dans la création d'emplois. Si au niveau national les repreneurs pourvoyeurs d'emplois sont principalement caractérisés par l'absence de contraintes au démarrage, aucune région française ne fait ressortir de manière significative cette association. En d'autres termes il n'y a pas de spécialisation régionale du modèle du repreneur non contraint pourvoyeur 
d'emplois. Une politique publique de l'emploi à l'échelle nationale par la création ou la reprise d'entreprises aurait a priori à court terme plus d'effets positifs en levant préférentiellement les contraintes sur les repreneurs même si elle ne concourrait pas nécessairement à la découverte et à l'exploitation de nouveaux marchés, de nouveaux produits... qui sont plutôt l'apanage des créateurs ex-nihilo.

Mots-clés : Créateur/repreneur d'entreprises, emplois, régions, méthodes d'analyse des données

JEL-code: C40, L10, L20 


\section{Introduction}

L'engagement dans un acte entrepreneurial est la concrétisation d'un projet économique dont l'espérance actualisée des gains anticipés par le créateur ou le repreneur excède la dépense initiale. Plus globalement le passage au statut de créateur ou de repreneur est la synthèse d'un engagement individuel et d'un environnement qui peut être plus ou moins favorable à l'entrepreneuriat (Shapero, 1982, Lacasse et Lambert, 1989). Le passage à l'acte d'entreprendre résulte donc d'une combinaison complexe de motivations (le goût d'entreprendre, le désir d'être indépendant etc.) et de contraintes (accès aux ressources financières, techniques, informationnelles etc.). Dans l'économie française, l'existence de contraintes à la création est souvent mise en avant pour justifier la faible propension à entreprendre. Si on considère le ratio des créateurs et des repreneurs d'entreprises rapporté à la population active, il était en 1997 de $75 \%$ plus élevé aux USA et 40\% plus élevé au Royaume-Uni qu'en France'.

Cette moindre propension à entreprendre est particulièrement pénalisante à l'heure où une part non négligeable des créations d'emplois est réalisée par les créateurs et les repreneurs d'entreprises. En 2002 les 218141 créateurs et repreneurs d'entreprises ont créé environ 458000 emplois même si la forte mortalité des nouvelles entreprises ${ }^{2}$ relativise la pérennité d'un certain nombre de ces emplois ${ }^{3}$.

Une plus forte propension à entreprendre couplée à une amélioration de la survie des nouvelles entreprises constituent un des moyens efficaces dans la recherche d'une plus grande création d'emplois pérennes ${ }^{4}$. Ces deux facteurs dépendent de l'environnement global de la création et en particulier de l'intensité et de la nature des contraintes (technique, informationnelle, d'insertion, financière) qui pèsent sur les créateurs et les repreneurs.

Cet environnement est aussi conditionné par la localisation géographique de la nouvelle entreprise. En effet, il existe des différences notables de développement entre les différentes régions françaises dues à un ensemble complexe de facteurs qui vont du renouvellement des activités motrices à l'attractivité des territoires en passant par l'existence de territoires de type "districts industriels". Ces territoires bénéficient alors de leur culture entrepreneuriale qui conditionne non seulement le modèle d'entrepreneurs (contraint/non contraint) mais aussi la pérennité et la croissance de l'entreprise créée. L'objet de ce papier est donc de rechercher dans quelle mesure les différentes régions françaises se regroupent ou s'opposent selon l'intensité de ces contraintes et s'il existe des spécificités régionales relativement au contenu en emplois véhiculé par les nouvelles entreprises.

Ce travail empirique est réalisé à partir du fichier SINE (Système d'Information sur les Nouvelles Entreprises) qui prend en compte les résultats d'une enquête effectuée par l'Institut

\footnotetext{
${ }^{1}$ En France, le déficit d'entrepreneurs est marquant puisqu'ils ne représentent que 3,2\% de la population adulte contre 5,16\% en Allemagne, 5,37\% au Royaume-Uni et 10,51\% aux Etats-Unis (Global Entrepreneurship Monitor [2002]).

${ }^{2}$ Dans cet article l'expression «nouvelles entreprises » inclue à la fois les créations ex-nihilo et les créations par reprise. Nous excluons les réactivations d'activité du champ de cette étude. De façon similaire l'expression « nouveaux entrepreneurs » correspond à l'ensemble des créateurs et des repreneurs.

${ }^{3}$ En France approximativement une entreprise sur deux cesse son activité avant sa cinquième année d'existence.

${ }^{4}$ A cet égard le gouvernement Raffarin a annoncé en juin 2002 son objectif de doubler le nombre de nouvelles entreprises afin d'atteindre un million d'emplois générés par les nouveaux entrepreneurs.
} 
National des Statistiques et des Etudes Economiques (INSEE). Notre échantillon comprend 21549 créateurs et repreneurs d'entreprises du premier semestre 1994 dont certains ont été ré-enquêtés en 1997. L'enquête de 1994 nous permet d'identifier les contraintes à la création et de rendre compte des différents profils de créateurs et de repreneurs dans les régions françaises. La réenquête de 1997 permet de connaître s'il y a échec entrepreneurial (disparition de l'entreprise du répertoire SIRENE -Système d'Informations et de Répertoire des Entreprises et des Etablissements-) ou non, et de caractériser pour les entrepreneurs toujours en activité l'évolution de l'effectif salarié entre 1994 et 1997. Nous séparons les individus qui créent ex nihilo de ceux qui créent par reprise pour identifier sur une base régionale les contraintes spécifiques à chacun de ces types et pour rechercher si le lien entre les contraintes à la création/reprise et le contenu en emplois des entreprises nouvelles diffère selon la modalité de l'acte entrepreneurial. Ce travail découle de l'interprétation des résultats d'enchaînements méthodologiques de différentes techniques d'analyse des données.

L'article est organisé de la manière suivante. La première section identifie les contraintes des créateurs et des repreneurs d'entreprises au regard de leur situation personnelle et sociale et de leur environnement. La deuxième section caractérise les profils représentatifs des créateurs et des repreneurs à partir des différentes contraintes repérées et associe à ces profils l'emploi généré par ces nouveaux entrepreneurs. La troisième section positionne, caractérise et éventuellement oppose des groupes de régions françaises selon les profils d'entrepreneurs (contraints/non contraints) et selon l'emploi généré par les nouveaux entrepreneurs. 


\section{I) LES DETERMINANTS DE l'EVENEMENT ENTREPRENEURIAL : DES CONTRAINTES SUBIES PAR LES CREATEURS-REPRENEURS A LEUR SITUATION PERSONNELLE ET SOCIALE}

Nous identifions un ensemble de caractéristiques personnelles et sociales significatives dans l'explication de l'acte entrepreneurial. Pour un certain nombre d'individus la disposition à l'action entrepreneuriale est expliquée non seulement par des caractéristiques psychologiques (le sentiment de pouvoir agir sur son devenir) mais elle est aussi induite par des trajectoires de vie (caractéristiques sociales qui touchent à la fois à des évènements de rupture personnelles, professionnelles et culturelles ...). Nous soulignons également le rôle joué par l'environnement du créateur et du repreneur en prenant en compte l'existence d'un entourage entrepreneurial et l'accès aux ressources nécessaires à la mise en œuvre du projet. Cet environnement est en partie déterminé par la localisation géographique du nouvel entrepreneur.

\section{1-1 ${ }^{\circ}$ ) Les fondements du passage à l'acte d'entreprendre :}

Les déterminants de l'acte entrepreneurial (cf. figure 1) résultent d'une combinaison entre des opportunités de créations-reprises d'entreprises et des contraintes liées à l'individu et à son environnement. Ces contraintes relèvent plus globalement du coût d'opportunité d'entreprendre (Storey, 1994). Ce coût renvoie d'une part à la situation personnelle et sociale du créateurrepreneur et, d'autre part, à l'existence d'un environnement plus ou moins favorable à la création (Shapero, 1975, 1982).

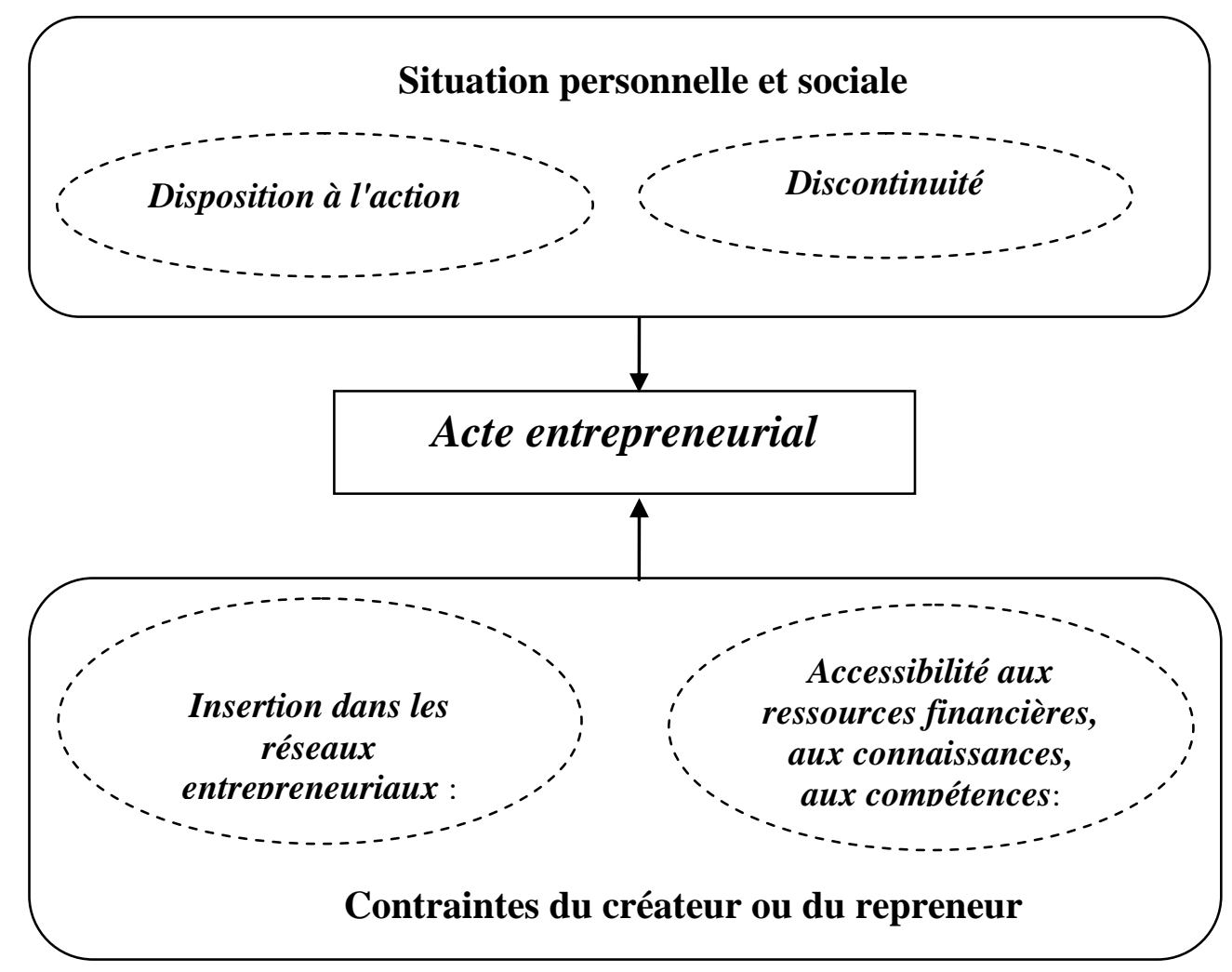

Figure 1 : Description de l'acte d'entreprendre 
La situation personnelle et sociale de l'individu est appréhendée à partir de caractéristiques personnelles déterminantes dans le passage à l'acte d'entreprendre. Ces caractéristiques individuelles sont reflétées par des variables qui non seulement caractérisent la disposition à l'action des individus mais qui également correspondent à des évènements de rupture au cours d'une vie.

La disposition à l'action de l'individu reflète le degré avec lequel il pense pouvoir agir sur son environnement. Dans la littérature psychologique, ce degré est connu sous le nom de "locus of control" (Rotter, 1966, 1971). Selon cette théorie, certains individus estiment que les récompenses qu'ils reçoivent dans la vie dépendent de forces indépendantes, en dehors de leur contrôle comme la chance, la bienveillance d'un mentor, ils sont peu entreprenants. En revanche d'autres pensent qu'ils sont en mesure de modifier le cours des événements et sont les plus susceptibles d'entreprendre. Un grand nombre d'études menées par des psychologues identifient cette population comme étant celle dont la principale motivation de la création/reprise d'entreprises est la volonté de maîtriser son propre destin (Hansemark, 2003).

La discontinuité dans la trajectoire de vie de l'individu résulte de deux sortes d'événements de rupture (Shapero, 1975): les événements positifs (pulls) et les événements négatifs (pushes). Les premiers correspondent à des créations/reprises liées à la confiance et au support d'un investisseur, à des opportunités de reprises ou à la découverte d'une opportunité nouvelle de marché. Les seconds correspondent à des décisions d'entreprendre liées à des chocs émotionnels (être divorcé, appartenir à une famille séparée etc...) ou à des évènements de rupture culturelle (personnes d'origine étrangère) ou sociale (perte d'emploi). L'intensité entrepreneuriale plus importante des chômeurs peut ainsi s'expliquer par la rupture dans la vie professionnelle de l'individu introduite par le passage à une situation temporaire d'inactivité. De même, la rupture personnelle liée à l'arrivée dans un nouveau pays joue sur la disposition à créer en raison de la recherche d'un statut social ou de la volonté de s'intégrer dans la société au moyen de l'entrepreneuriat. On peut citer à titre d'exemples de vagues d'entrepreneurs les cas des pieds noirs français qui ont quitté l'Algérie, des réfugiés cubains qui ont quitté Cuba pour les Etats-Unis, plus récemment des réfugiés Vietnamiens des boat-people... Ainsi, les réfugiés constituent une force entrepreneuriale importante que l'on retrouve historiquement dans un grand nombre de pays. A cet égard, Hagen (1962) a montré que la propension à entreprendre parmi certains groupes de population peut être expliquée par le fait qu'ils ont été dépossédés de leur statut social. Cette dépossession crée de l'anxiété, des frustrations et le désir de changer leur situation se manifeste par l'entrepreneuriat.

La présence d'un entourage entrepreneurial et un accès aisé aux ressources nécessaires à la mise en œuvre d'une entreprise caractérise l'intensité des différentes contraintes auxquelles fait face un créateur ou un repreneur d'entreprise.

L'insertion dans les réseaux entrepreneuriaux résulte de la présence d'un entourage entrepreneurial qui est plus ou moins marqué dans les territoires selon l'existence d'une culture entrepreneuriale. Cette insertion favorise naturellement le passage à l'acte d'entreprendre, crédibilise l'acte de la création et permet parfois de bénéficier d'un support financier familial.

L'accessibilité aux ressources concerne non seulement les ressources financières, mais aussi l'accès aux connaissances, aux compétences, au savoir-faire, à l'information... 
Ces différents "ingrédients" du passage à l'acte d'entreprendre ne sont pas indépendants et entretiennent des relations de complémentarité ou de substituabilité. Ainsi, l'insertion dans les réseaux entrepreneuriaux favorise en général l'accès aux ressources (relation de complémentarité). En revanche, une disposition à l'action relativement faible peut être compensée par le fait que l'individu appartient à une famille d'entrepreneurs ou bénéficie d'une culture entrepreneuriale (relation de subsituabilité ${ }^{5}$ ).

Le passage à l'acte d'entreprendre est la résultante d'une combinaison complexe entre la situation personnelle et sociale d'un individu et les contraintes liées à la création/reprise. L'intensité et la nature de ces contraintes peuvent être mises en perspective avec la localisation géographique de l'entreprise nouvellement créée ou reprise.

\section{$\left.1-2^{\circ}\right)$ La dimension spatiale de l'acte d'entreprendre :}

L'insertion dans les réseaux entrepreneuriaux est facilitée par la présence d'une culture entrepreneuriale plus ou moins marquée selon les territoires. A une échelle infrarégionale, cette culture entrepreneuriale fait notamment référence à l'existence de districts industriels dans lesquels la fonction entrepreneuriale est valorisée (ce qui se traduit par une intensité entrepreneuriale plus importante). Dans le cas français, les districts industriels ne représentent pas une organisation territoriale aussi développée qu'en Italie et beaucoup de ces districts ont perdu de leur importance en raison du déclin de leur spécialité de base (coutellerie de Thiers, bonneterie à Roanne, ganterie de Millau (Carluer, 1998)). Cependant, dans la période récente, le Choletais et le Nord-Est vendéen représentent une organisation territoriale qui, bien que spécialisée sur des activités en déclin, se montre plus performante que ce que sa spécialité supposerait. Son succès fondé sur "l'usine à la campagne" a permis à ce territoire de se diversifier et a contribué dans la période récente au "dynamisme" de la région des Pays de la Loire.

L'accès aux ressources entretient des relations complexes avec l'espace.

En ce qui concerne les ressources financières, Cieply (2001) met en évidence l'existence de vides de financement en fonds propres dans certaines zones du territoire, rurales, plus particulièrement pour des projets de taille médiane pour les nouvelles activités. Dans une étude récente, Guiso, Sapiensa et Zingales (2002), montrent que l'existence de structures de financement locales dans les différentes régions italiennes accroît la probabilité qu'un individu créé une entreprise.

L'accès aux connaissances est a priori favorisé dans les milieux innovateurs (technopoles) qui se caractérisent par la concentration d'entreprises de haute technologie dans certains lieux et qui sont aussi des lieux favorisant la création d'entreprises ${ }^{6}$. Par ailleurs, l'accès aux connaissances est sensible au degré d'urbanisation de la région qui permet de bénéficier d'économies externes d'agglomération (facilité d'accès aux études, aux conseils ..).

\footnotetext{
${ }^{5}$ Dans le même ordre d'idées, une disposition à l'action relativement faible peut-être compensée par la discontinuité induite par le fait d'être au chômage. Inversement une disposition à l'action forte peut-être contrainte par les difficultés d'accès aux ressources que subit l'entrepreneur.

${ }^{6}$ On retrouve comme espace fonctionnant selon le principe technopolitain la ZIRST de Meylan dans la région RhôneAlpes, Orsay-Saclay-Les Ulis dans la région Île-de-France, Sophia-Antipolis dans la région Provence-Alpes-Côted'Azur.
} 
L'accès aux compétences techniques est facilité par une expérience professionnelle qui serait acquise dans le même secteur d'activité que celui de l'entreprise nouvellement créée. Dans le cas de régions de vieille industrie en reconversion, les nouveaux entrepreneurs souffrent d'une mauvaise spécialisation dans la mesure où cette dernière est acquise dans des secteurs en crise et le plus souvent dans des entreprises de taille importante (ce qui peut nuire à la perception du fonctionnement global de l'entreprise et à la fonction entrepreneuriale).

La situation personnelle et sociale globale de l'individu est dans une moindre mesure dépendante de la localisation de la nouvelle entreprise. En ce qui concerne la situation personnelle, ce sont avant tout les traits de personnalité de l'entrepreneur qui importent. En ce qui concerne la situation sociale, la discontinuité dans la vie professionnelle que constitue le chômage est difficile à interpréter au niveau régional. En effet, la relation entre le taux de chômage et l'intensité entrepreneuriale à l'échelle régionale est ambiguë puisque l'on peut distinguer à l'échelon régional un chômage de reconversion (dans les régions en crise) qui suscite peu d'activité entrepreneuriale et un chômage de croissance (dans les régions attractives) qui est porteur d'un certain dynamisme entrepreneurial.

\section{II) QUELS PROFILS DE CREATEURS OU DE REPRENEURS SONT POURVOYEURS D'EMPLOIS ?}

Les méthodes de l'analyse des données nous permettent d'identifier la nature des contraintes pesant sur les créateurs et les repreneurs et de déduire de celles-ci les profils types de ces deux populations d'entrepreneurs. En croisant ces profils avec le contenu en emplois de ces entreprises nous montrons que l'absence de contraintes au démarrage caractérise généralement les nouveaux entrepreneurs pourvoyeurs d'emplois mais de manière plus prononcée pour les repreneurs que pour les créateurs.

\section{2-1) La base de données et la construction des variables :}

La base de données utilisée provient du dispositif SINE qui rend compte, par le biais d'enquêtes directes menées par l'INSEE, du profil de l'entrepreneur, du contexte et de l'environnement de la création-reprise d'entreprises françaises au cours du premier semestre 1994. A partir d'un échantillon initial de 30778 créateurs ou repreneurs représentatifs de la totalité des créateurs ou repreneurs d'entreprises du premier semestre 1994 (soit 96407 créateurs ou repreneurs à l'échelon national ${ }^{7}$ ), nous avons retenu 25682 entrepreneurs. Nous avons exclu de notre échantillon les filiales et les projets d'équipe ce qui nous permet d'associer chaque entreprise créée ou reprise à un individu. Ces entreprises privées relèvent de l'industrie, de la construction, $\mathrm{du}$ commerce et des services (exclusion des secteurs agricole et financier). Compte tenu de l'élimination des départements d'outre mer et des entrepreneurs qui n'indiquaient pas leur effectif salarié en 1994, notre échantillon comprend finalement 21549 créateurs ou repreneurs (soit 63637 entrepreneurs lorsque l'échantillon est redressé : 48751 créateurs et 14886 repreneurs).

\footnotetext{
7 Les unités ont été redressées selon la terminologie de l'Insee par hot-deck (avec croisement de l'origine de l'entreprise, de la localisation régionale et de l'activité en 9 postes) et par repondération.
} 
Nous sélectionnons les questions de l'enquête de manière à rendre compte de la situation personnelle et sociale de l'individu et d'un environnement plus ou moins favorable à la création (cf. annexe1).

En séparant la population des créateurs de celle des repreneurs, l'objectif poursuivi ici est de décrire les principales contraintes qui pèsent sur les créateurs et les repreneurs et de rendre compte de l'évolution de l'emploi qu'ils génèrent. La situation personnelle et sociale de l'entrepreneur ainsi que le secteur d'activité de la nouvelle entreprise sont positionnés en variables supplémentaires et illustratives qui viennent a posteriori enrichir cette description. Les méthodes d'analyse des données nous permettent également de positionner les 22 régions françaises ${ }^{8}$ de les répartir en classes homogènes selon les deux précédents critères mais également d'établir une liaison entre les contraintes à la création et le contenu en emplois véhiculé par ces nouvelles entreprises (cf. figure 2).

Les analyses proposées sont basées sur la mise en œuvre conjointe de méthodes factorielles en l'occurrence l'Analyse des Correspondances Simples et Multiples (Benzécri, 1973) et de Classifications Hiérarchiques Ascendantes consolidées par la méthode des centres mobiles (Diday, 1971).

\footnotetext{
${ }^{8}$ L'enquête SINE ne permet pas d'obtenir des informations statistiquement fiables à un niveau infra-régional.
} 


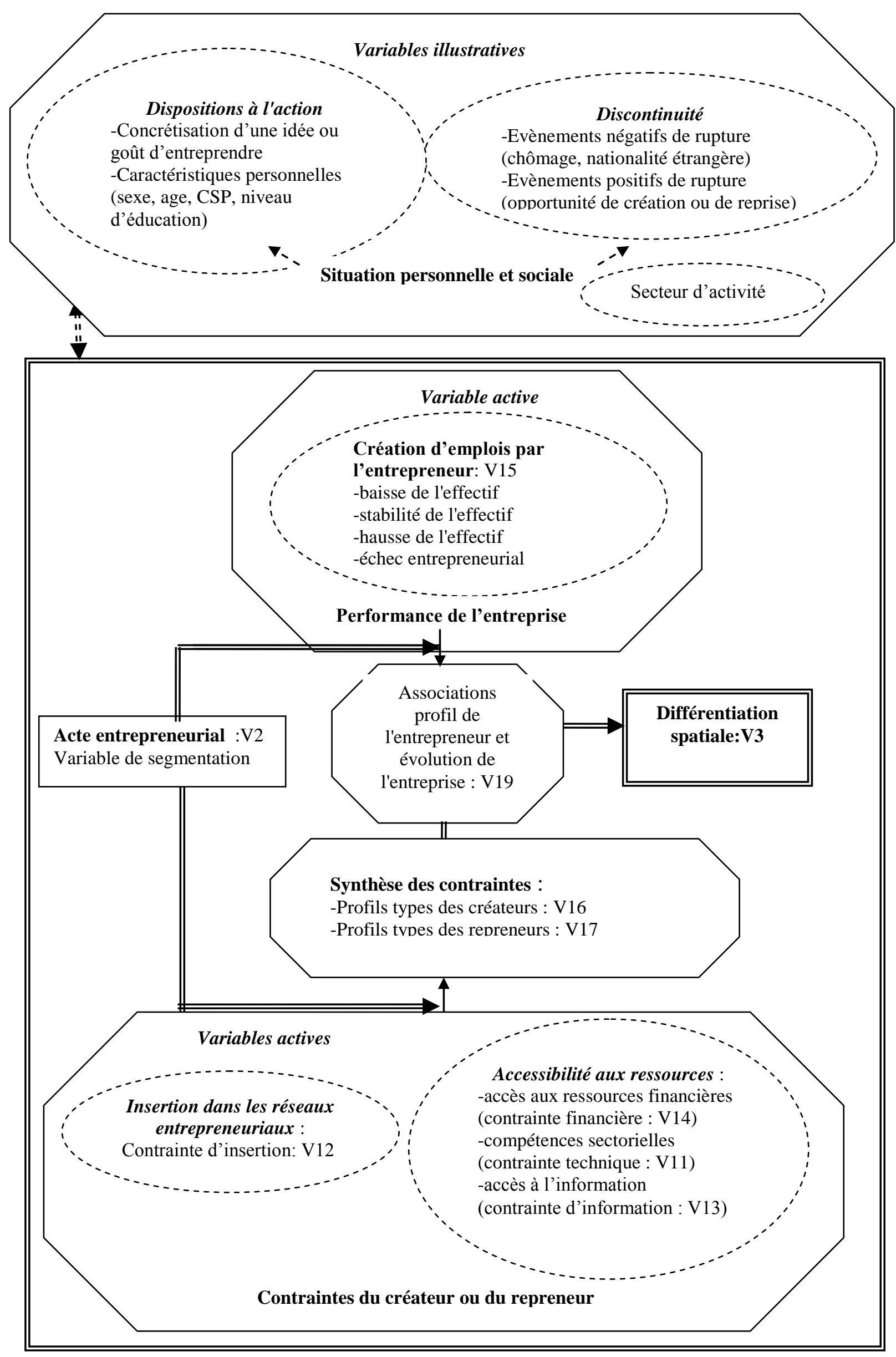

Figure 2 : la transcription des contraintes et des incitations liées à l'acte d'entreprendre 
La situation personnelle et sociale de l'individu est synthétisée par des variables représentatives de sa disposition à l'action et de phénomènes de discontinuité.

La disposition à l'action est caractérisée à partir des cinq variables suivantes :

-Les deux modalités "nouvelle idée" et "goût d'entreprendre" en réponse à la question "Quelles sont vos principales motivations dans le passage à l'acte d'entreprendre ?" constituent de bonnes indicatrices de la disposition à 1 'action (variable $\mathrm{n}^{\circ} 10$ relative aux "motivations de l'acte entrepreneurial").

-Les variables "sexe", "âge", "catégorie socioprofessionnelle" et "niveau d'éducation" (variables $\mathrm{n}^{\circ} 4,5,6$ et 8 ) sont prises en compte dans la mesure où il existe une différence notable d'intensité entrepreneuriale (cf. annexe 2) entre les hommes et les femmes, selon la tranche d'âge, en fonction du précédent travail et selon le niveau de diplôme (variable représentative du capital humain initial de l'entrepreneur).

La discontinuité est reflétée par trois variables :

-Variable "activité préalable" (variable $n^{\circ} 7$ ): elle prend en compte le statut de l'entrepreneur avant son passage à l'acte (était-il chômeur, actif ou inactif ?). Cette variable est particulièrement pertinente car plus de $40 \%$ des nouveaux entrepreneurs lors de l'enquête Sine 1994 étaient d'anciens chômeurs ${ }^{9}$.

-Variable "nationalité" (variable $n^{\circ}$ ) : à partir de l'enquête Sine, nous pouvons constater que la population d'origine étrangère a une intensité entrepreneuriale qui est 2,5 fois plus importante que dans la population d'origine Française ${ }^{10}$. Le rôle joué par la création d'entreprise en tant que mode d'insertion professionnel des immigrés est manifeste dans l'économie française : en 2000, 7,1\% des artisans, commerçants et chefs d'entreprises étaient d'origine étrangère contre $5,1 \%$ en 1989 .

-Les deux modalités "Sans-emploi"11 et "Opportunité" de la variable "motivations de l'acte entrepreneurial" (variable $\mathrm{n}^{\circ} 10$ ) rendent compte respectivement d'une discontinuité "négative" et "positive" (Shapero, 1975).

Ces variables seront retenues comme des variables illustratives. Elles sont cependant pertinentes car elles influent sur l'intensité entrepreneuriale (cf. annexe 2) bien que leur lien avec la localisation des entreprises soit plus difficile à établir. Dans notre approche statistique, ces variables viendront donc illustrer les contraintes à la création/reprise définies à partir des caractéristiques sur l'environnement. Pour tenir compte de la disparité des dynamiques

\footnotetext{
${ }^{9}$ On notera cependant qu'il existe une dimension conjoncturelle non négligeable dans ce résultat (conséquence de la crise de 1993). Lors de l'enquête sur la nouvelle cohorte des créateurs-repreneurs de 1998, la proportion de chômeurscréateurs n'était que de $28 \%$.

${ }^{10}$ Cette caractéristique est à la fois la résultante des difficultés d'intégration mais elle rend compte aussi de l'existence de réseaux ethniques qui peuvent favoriser l'installation des nouveaux entrepreneurs.

${ }^{11}$ On pourrait penser que les modalités «sans emploi » de la variable $\mathrm{n}^{\circ} 10$ « motivation » et chômage de la variable $\mathrm{n}^{\circ} 7$ « activité préalable » sont redondantes. Cependant il se peut qu'une partie des chômeurs créateurs ou repreneurs ait eu comme motivation principale soit une nouvelle idée ou le bénéfice d'une opportunité voire le goût d'entreprendre (le chômage peut-être l'occasion de mettre en œuvre un projet qui a été longtemps mûri mais qui n'a pu trouver son aboutissement tant que l'individu était salarié). De plus sur un plan purement statistique ces modalités de deux variables différentes relèvent de la catégorie des variables illustratives et sont à ce titre projetées une à une et de façon indépendante sur les axes principaux lors des analyses factorielles des correspondances et n'interviennent en aucun cas dans la détermination des classes obtenues lors de la classification hiérarchique ascendante.
} 
entrepreneuriales selon l'activité de l'entreprise, nous avons ajouté au titre des variables illustratives le secteur d'activité de l'entreprise (variable ${ }^{\circ} 20$ ).

La nature des contraintes subies par le créateur ou le repreneur rend compte de l'environnement plus ou moins favorable à l'acte entrepreneurial ${ }^{12}$. Nous retenons les quatre contraintes suivantes (cf. annexe 3 pour le mode exact d'identification de ces contraintes à partir de l'enquête SINE) :

-Variable "contrainte d'insertion" (variable $\mathrm{n}^{\circ} 12$ ) : elle rend compte du degré d'insertion du créateur ou du repreneur dans les réseaux entrepreneuriaux et de la dimension reproduction sociale dans l'acte entrepreneurial. Cette variable est particulièrement pertinente dans l'explication de la pérennité des entreprises créées ou reprises : les entrepreneurs insérés dans les réseaux entrepreneuriaux bénéficient généralement du support financier de la famille et donc appartiennent à des entreprises de plus grandes classes de taille (Volery et Servais, 2001). De plus, une bonne insertion dans les réseaux peut permettre de contracter avec un «bon» fournisseur ou un «bon» client : elle contribue à convaincre de travailler avec l'entreprise nouvellement créée ou reprise et crédibilise tout accord noué avec cette dernière. Pour ces multiples raisons, l'insertion favorise la durée de vie de l'entreprise (Gollac et Lauhle, 1987 ; Abdesselam, Bonnet et Le Pape, 2004).

-Variable "contrainte technique" (variable $\mathrm{n}^{\circ} 11$ ) : cette variable est construite de manière telle que le créateur ou le repreneur est supposé disposer de compétences techniques d'autant plus affirmées qu'il a lui-même acquis son expérience dans un secteur identique ou proche de celui de l'entreprise nouvellement créée ou reprise. Cette variable est représentative du capital humain acquis par l'expérience. Cette variable est importante car la connaissance du secteur est primordiale pour la réussite de l'entreprise (Abdesselam, Bonnet et Le Pape, 2004).

-Variable "contrainte financière" (variable $n^{\circ} 14$ ) : l'existence d'une contrainte financière pour le créateur ou le repreneur est repérée par croisement de plusieurs questions de l'enquête. Si l'entrepreneur a demandé un prêt bancaire et ne l'a pas obtenu, nous considérons qu'il est contraint financièrement. Nous avons aussi pris en compte le phénomène d'auto exclusion du marché du crédit bancaire $^{13}$, en supposant que les entrepreneurs qui ne demandent pas de prêts bancaires pour de petits projets d'investissements (moins de 3811 Euros) peuvent s'autoexclure du marché du crédit et pour cette raison mettent en place des projets de dimension modeste ${ }^{14}$ (d'où l'existence d'une contrainte financière pour cette catégorie d'individus). Cette contrainte renvoie au phénomène du rationnement du crédit bancaire qui affecte les nouvelles entreprises (Cieply et Paranque, 1998, Crépon et Duguet, 2002). Ces derniers font ressortir les effets négatifs du rationnement du crédit sur la survie des nouvelles entreprises à partir de l'enquête SINE.

Les difficultés d'accès au prêt bancaire liées au manque de confiance des banquiers vis-àvis des créateurs ont un rôle décisif dans la pérennité des entreprises : les entreprises qui ont moins

\footnotetext{
${ }^{12}$ Dans le cas français, cet environnement se traduit parfois par des obstacles à la création d'entreprises. Ainsi, une récente étude APCE-IFOP (avril 1998) montre que trois obstacles majeurs jouent sur la faible propension à créer ou reprendre : le faible esprit entrepreneurial, le manque d'accompagnement des créateurs et les difficultés financières.

${ }^{13}$ qui se traduit par une proportion significativement plus importante d'entreprises mortes au bout de 4 ans parmi celles qui n'ont pas demandé de prêt bancaire.

${ }^{14}$ A priori ceux qui ne demandent pas de prêts bancaires pour des projets plus importants ne subiraient pas de contrainte financière liée à l'autoexclusion.
} 
de 1500 euros au départ ont un taux de survie à 5 ans de 39\%, ce taux est doublé selon l'Agence pour la Création d'Entreprises (APCE) si la somme initiale est supérieure à 150000 euros.

-Variable "contrainte informationnelle" (variable $\mathrm{n}^{\circ} 13$ ): elle est construite par la combinaison de réponses données à deux questions portant sur la réalisation d'études ou d'enquêtes avant la création ou la reprise et sur la prise de contacts initiaux avec des clients potentiels. Cette variable est représentative de la démarche de l'individu en matière d'acquisition d'informations relatives à la faisabilité ou au potentiel du projet. Si l'individu ne réalise ni études, ni enquêtes et ne prend pas de contact avec des clients, nous interprétons ces réponses comme étant le signe d'une contrainte informationnelle que l'individu s'est en partie lui-même créé et qui résulte peut-être d'une plus faible disposition à l'action.

Dans la période précédant l'acte entrepreneurial, l'insuffisance de l'information et du conseil (sur les produits, le marché, les clients...) constitue un handicap majeur dans le choix de la localisation de l'entreprise créée et/ou de son aire de marché. Ainsi, selon 1' APCE 70\% des faillites d'entreprises seraient imputables à l'absence ou à la mauvaise qualité des études de marché préalables.

Pour obtenir des profils d'entrepreneurs, nous retenons la variable « acte entrepreneurial » (variable $\mathrm{n}^{\circ} 2$ ) en séparant les deux populations : les créateurs et les repreneurs. Nous caractérisons ces deux populations en fonction de deux groupes de variables :

-les variables représentatives des contraintes à la création/reprise (variables $n^{\circ} 11$ à $n^{\circ} 14$ ).

-la variable «création d'emplois par l'entrepreneur» (variable $\mathrm{n}^{\circ} 15$ ) : on considère quatre états possibles de l'entreprise en 1997 : sa disparition (qui caractérise un échec entrepreneurial), une baisse, une stabilité ou une hausse de son effectif salarié (pour les entreprises toujours en activité en 1997). Cette variable permettra d'identifier la nature de la relation entre d'une part les contraintes qui pèsent sur les créateurs et les repreneurs et d'autre part le contenu en emploi de l'entreprise nouvellement créée ou reprise. D'autres facteurs relevant de la stratégie de développement de l'entreprise ont une influence sur sa politique de l'emploi ${ }^{15}$. En particulier le comportement de l'entrepreneur sur son marché (son agressivité) est lié à la pérennité de l'entreprise (Bonnet, Gharbi Fessi et Le Pape, 2003) ${ }^{16}$. Cependant l'agressivité correspond à la politique courante de l'entreprise alors que nous mettons l'accent ici sur le rôle joué par le contexte initial de la création-reprise d'entreprises.

En résumé, nous partons de l'analyse de Shapero (1975) pour justifier le choix de nos variables considérées comme étant des déterminants majeurs du passage à l'acte d'entreprendre (et plus particulièrement l'identification de contraintes à la création-reprise). Nous enrichissons cette approche en construisant des profils de créateurs et de repreneurs d'entreprises (variables $n^{\circ} 16$ et $\mathrm{n}^{\circ} 17$ ) et en recherchant les éléments d'une différenciation spatiale (variable $n^{\circ} 3$ ) des profils de ces deux populations d'entrepreneurs et les performances en création d'emplois des différents types de créateurs/repreneurs (variable $n^{\circ} 15$ ).

\section{2-2) La caractérisation des profils des créateurs et des repreneurs d'entreprises :}

\footnotetext{
${ }^{15}$ Nous remercions un des rapporteurs de nous avoir suggéré cet aspect.

${ }^{16}$ L'agressivité est mesurée à partir de la politique de prix, les dépenses publicitaires, la prospection de nouveaux clients, la volonté d'accroître le chiffre d'affaires et les travaux de sous-traitance donnés. Au moyen d'un modèle de durée, nous montrons que l'échec entrepreneurial peut être imputable à une faible agressivité de l'entrepreneur.
} 
Indépendamment de l'espace nous caractérisons les créateurs et les repreneurs selon les contraintes qu'ils subissent. Chaque variable de segmentation a fait alors l'objet d'un test non paramétrique d'homogénéité afin d'examiner comment se répartissent les entrepreneurs de chacune des deux populations (créateurs et repreneurs) suivant les modalités de chacune des quatre contraintes et de l'effectif salarié. On constate alors que le poids des contraintes se répartit différemment entre les créateurs et les repreneurs. De même, la création d'emplois des créateurs purs diffère de celle des repreneurs (cf tableau 1).

\begin{tabular}{|c|c|c|c|}
\hline \multicolumn{2}{|c|}{$\begin{array}{l}\text { Variables de segmentation } \\
\text { Contraintes et création d'emplois par } \\
\text { l'entrepreneur }\end{array}$} & $\begin{array}{l}\text { En pourcentage dans la } \\
\text { population des créateurs }\end{array}$ & $\begin{array}{l}\text { En pourcentage dans la } \\
\text { population des repreneurs }\end{array}$ \\
\hline \multirow{4}{*}{$\begin{array}{c}\text { CONTRAINTES } \\
\text { AU } \\
\text { DEMARRAGE* }\end{array}$} & $\begin{array}{c}\text { Contrainte technique } \\
\text { décelée }\end{array}$ & $35,37 \%+$ & $34,34 \%$ \\
\hline & $\begin{array}{c}\text { Contrainte d'insertion } \\
\text { décelée }\end{array}$ & $28 \%+$ & $27,05 \%$ \\
\hline & $\begin{array}{c}\text { Contrainte } \\
\text { informationnelle décelée }\end{array}$ & $20,53 \%$ & $32,22 \%+$ \\
\hline & $\begin{array}{c}\text { Contrainte financière } \\
\text { décelée }\end{array}$ & $33,04 \%+$ & $19,92 \%$ \\
\hline \multirow{4}{*}{$\begin{array}{l}\text { CREATION } \\
\text { D'EMPLOIS } \\
\text { (1994-1997) }\end{array}$} & $\begin{array}{l}\text { Baisse de l'effectif } \\
\text { salarié }\end{array}$ & $7,00 \%$ & $13,70 \%+$ \\
\hline & $\begin{array}{c}\text { Stabilité de l'effectif } \\
\text { salarié }\end{array}$ & $26,88 \%$ & $29,48 \%+$ \\
\hline & $\begin{array}{c}\text { Hausse de l'effectif } \\
\text { salarié }\end{array}$ & $19,12 \%$ & $22,46 \%+$ \\
\hline & Echec entrepreneurial & $47,00 \%+$ & $34,36 \%$ \\
\hline
\end{tabular}

*Un entrepreneur peut subir plusieurs contraintes à la fois, les contraintes ne sont pas exclusives.

Le signe + localise la disparité et signifie que l'écart entre les proportions de chaque population est significatif avec un risque d'erreur inférieur ou égal à $5 \%$.

\section{Tableau 1 : poids des contraintes et évolution de l'effectif selon les modalités de l'acte entrepreneurial}

A la lumière de ces résultats, l'hypothèse d'homogénéité est rejetée. Relativement aux repreneurs, les créateurs d'entreprises sont globalement plus contraints ${ }^{17}$. En effet la contrainte technique, la contrainte d'insertion dans les réseaux entrepreneuriaux et surtout la contrainte financière les affectent plus spécifiquement. En revanche, la contrainte informationnelle touche davantage les repreneurs (l'existence d'un marché préalable semble dispenser certains d'entre eux d'effectuer des études, des enquêtes avant la reprise). De plus, la proportion d'échec entrepreneurial à quatre ans est significativement plus importante dans la population des créateurs.

\footnotetext{
${ }^{17}$ Une proportion relativement faible de créateurs et de repreneurs de notre échantillon sont contraints (de $1 / 5$ à environ 1/3) et ce pour les quatre types de contraintes retenues. La prise en compte des secteurs d'activité montre que les créateurs les plus contraints relèvent du secteur des services aux ménages et les moins contraints appartiennent à l'industrie, à la construction et aux services aux entreprises. Les repreneurs les moins contraints appartiennent aux services aux ménages et les plus contraints au commerce.
} 
Une étude séparée se justifie en raison du profil différencié des créateurs et des repreneurs à la fois selon la nature des contraintes qu'ils subissent et selon l'évolution de la création d'emplois qu'ils génèrent.

Nous avons alors effectué une classification sur les créateurs et une autre classification sur les repreneurs selon les contraintes qu'ils subissent. Ces classifications permettent de caractériser des profils types de créateurs et des profils types de repreneurs à partir des seize combinaisons possibles des contraintes initiales (tableau 2). La méthode utilisée est un enchaînement méthodologique d'une Classification Hiérarchique Ascendante (CHA) appliquée aux facteurs significatifs de l'Analyse Factorielle des Correspondances Multiples (AFCM) des contraintes (cf annexe 4). Nous avons retenu une partition en quatre classes, ce qui nous permet de définir quatre principaux profils types de créateurs et quatre principaux profils types de repreneurs.

\begin{tabular}{|c|c|c|c|c|c|}
\hline & Types d'entrepreneur* & $\begin{array}{c}\text { Contrainte } \\
\text { technique }\end{array}$ & $\begin{array}{l}\text { Contrainte } \\
\text { d'insertion }\end{array}$ & $\begin{array}{c}\text { Contrainte } \\
\text { informationnelle }\end{array}$ & $\begin{array}{l}\text { Contrainte } \\
\text { financière }\end{array}$ \\
\hline \multirow{4}{*}{ 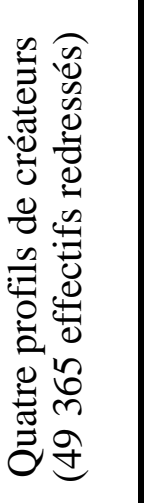 } & $\begin{array}{l}\text { Créateur non contraint } \\
(43 \%)\end{array}$ & Non décelée & Non décelée & Non décelée & Non décelée \\
\hline & $\begin{array}{c}\text { Créateur isolé } \\
(19,94 \%)\end{array}$ & Non décelée & Décelée & Non décelée & Non décelée \\
\hline & $\begin{array}{c}\text { Créateur non expérimenté et } \\
\text { contraint financièrement } \\
(16,52 \%)\end{array}$ & Décelée & Non décelée & Non décelée & Décelée \\
\hline & $\begin{array}{c}\text { Créateur contraint } \\
(20,53 \%)\end{array}$ & Décelée & Décelée & Décelée & Décelée \\
\hline \multirow{4}{*}{ 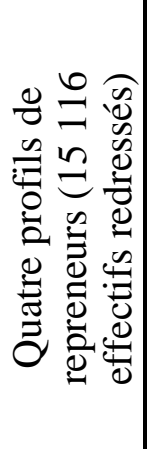 } & $\begin{array}{c}\text { Repreneur non contraint } \\
(44,36 \%)\end{array}$ & Non décelée & Non décelée & Non décelée & Non décelée \\
\hline & $\begin{array}{c}\text { Repreneur non informé } \\
(13,58 \%)\end{array}$ & Non décelée & Non décelée & Décelée & Non décelée \\
\hline & $\begin{array}{l}\text { Repreneur isolé et non } \\
\text { expérimenté }(22,14 \%)\end{array}$ & Décelée & Décelée & Non décelée & Non décelée \\
\hline & $\begin{array}{l}\text { Repreneur contraint mais } \\
\text { inséré }(19,92 \%)\end{array}$ & Décelée & Non décelée & Décelée & Décelée \\
\hline
\end{tabular}

*Résultats AFCM+CHA

Tableau 2 : Caractérisation des profils types des créateurs et des profils types des repreneurs à partir des contraintes.

On retrouve le profil de l'entrepreneur non contraint sur chacune de nos deux souspopulations. L'introduction de variables illustratives permet d'enrichir la caractérisation des profils types. Ainsi, le profil type du repreneur non contraint, à la différence de celui des créateurs non contraints, correspond à des hommes, dont l'âge est relativement jeune (moins de 30 ans) et dont la catégorie socioprofessionnelle avant la création intègre les ouvriers et les employés dans les secteurs des services aux entreprises et aux ménages (alors que les créateurs non contraints sont plutôt des cadres, des chefs d'entreprises et des agents de maitrise dans les secteurs de l'industrie, des transports et du commerce). Une différence notable sur les motivations de l'acte entrepreneurial apparaît : le goût d'entreprendre (modalité représentative de la disposition à l'action 
selon Shapero) ressort plus dans la population des repreneurs non contraints, en revanche, la nouvelle idée, l'exemple de l'entourage et l'opportunité (modalités représentatives d'un évènement positif de discontinuité selon Shapero) caractérisent davantage la motivation des créateurs non contraints.

En ce qui concerne les créateurs contraints, à la différence des créateurs non contraints, les variables supplémentaires relatives à leur situation personnelle et sociale montrent que ces derniers sont le plus souvent non diplômés (le créateur ou le repreneur non contraint étant diplômé), de nationalité étrangère (le créateur ou le repreneur non contraint étant de nationalité française) et motivent la création par l'absence d'emploi (évènement négatif de discontinuité selon Shapero). On les trouve plus spécifiquement dans les secteurs des services aux ménages.

\section{2-3) Création d'emplois et profils des créateurs et des repreneurs :}

Une typologie des créations d'emplois selon les profils des créateurs purs montre que les entrepreneurs qui créent des emplois et ceux qui en détruisent ont comme unique profil caractéristique celui du créateur non contraint (cf. figure 3). La méthode statistique multidimensionnelle utilisée à chacune de nos deux sous-populations est une CHA appliquée aux facteurs significatifs d'une Analyse Factorielle des Correspondances (AFC) croisant la création d'emplois et les contraintes liées à l'acte entrepreneurial. Les résultats de cette analyse montrent que les créateurs qui ont subi un échec entrepreneurial appartiennent à deux profils types d'entrepreneurs contraints.

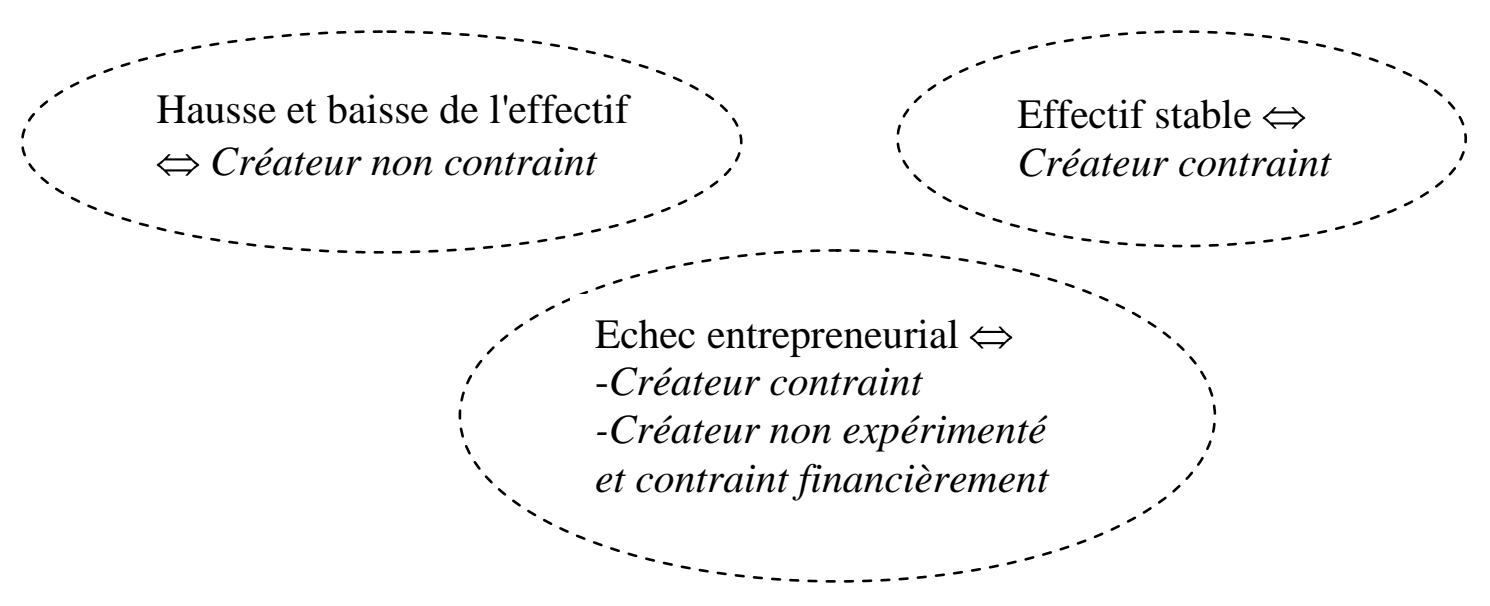

Résultats AFC+CHA

Figure 3 : Associations profils types de créateurs et création d'emplois.

On observe donc une liaison assez ambiguë entre le poids des contraintes et la création d'emplois par les créateurs : si une atténuation des contraintes au démarrage est liée à la réussite entrepreneuriale (modèle des créateurs non contraints), ce modèle de créateurs se retrouve non seulement sur la catégorie des entrepreneurs qui ont créé des emplois mais aussi sur ceux qui en ont détruit. Une interprétation possible réside peut-être dans le fait que l'absence de contraintes permet une plus grande latitude de l'entrepreneur dans sa prise de risque, ce qui peut se traduire par une variabilité accrue de la taille de l'entreprise. En revanche, pour le modèle du créateur 
contraint, la stratégie de croissance de l'entreprise semble «bridée ». L'introduction des variables illustratives sur les associations entre les profils de créateurs et la création d'emplois enrichit l'interprétation de ces associations (cf annexe 5). On constate alors que les deux modèles de créateurs liés à l'échec entrepreneurial correspondent à des individus disposant d'un faible capital humain initial (non diplômés) et dont la motivation peut aussi refléter des difficultés d'intégration (population étrangère, chômeurs). Ces associations se retrouvent plus dans les secteurs des hôtelscafés-restaurants et du commerce. L'association sur le modèle du créateur non contraint relève d'un type d'entrepreneur disposant d'un capital humain élevé (bac plus deux et plus) et dont la disposition à l'action correspond au goût d'entreprendre, de nationalité française et actif (donc a priori pouvant bénéficier des relations issues de leur expérience professionnelle actuelle). Les secteurs concernés par cette association correspondent à l'industrie et à la construction.

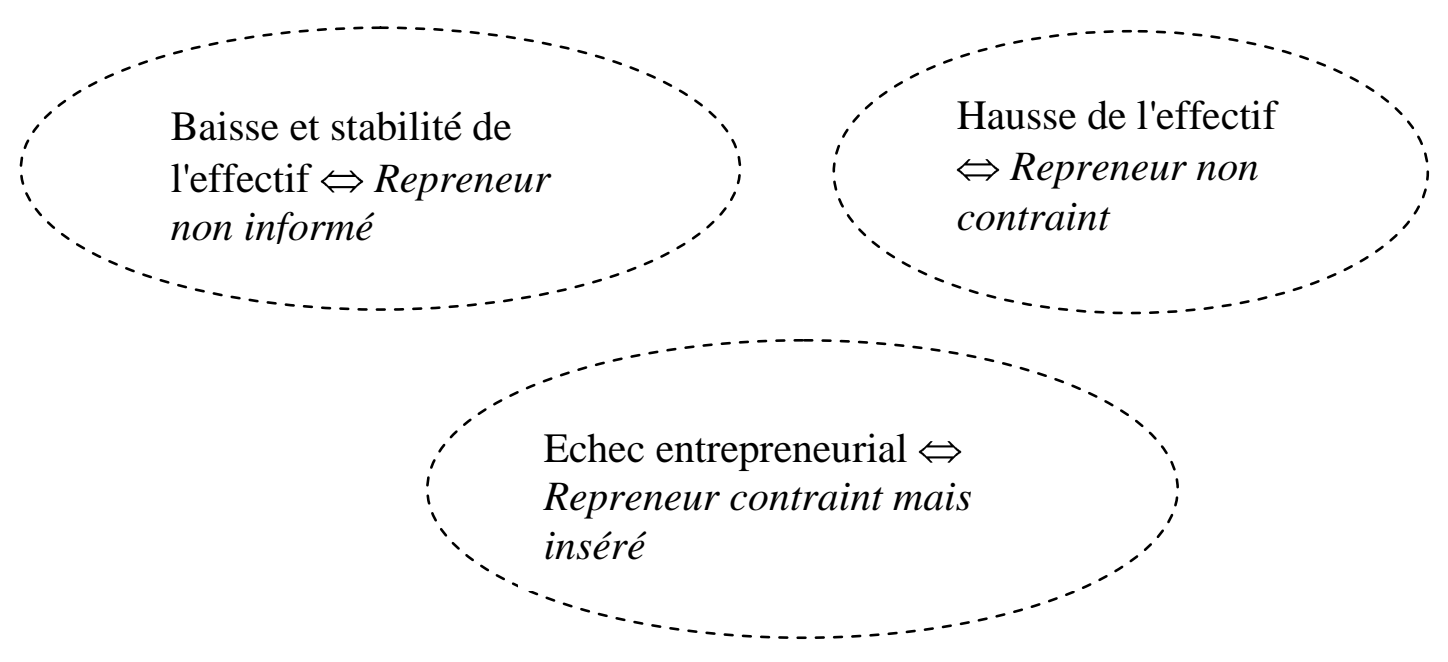

Résultats AFC+CHA

Figure 4 : Associations profils types de repreneurs et création d'emplois.

Les résultats de ces associations montrent une opposition entre l'échec entrepreneurial et les autres classes de créations d'emplois relativement au profil des repreneurs. Parmi les repreneurs toujours en activité en 1997, l'opposition renvoie à ceux qui ont créé des emplois entre 1994 et 1997 par rapport aux deux autres classes (baisse et stabilité de l'effectif). A la différence de la population des créateurs, celle des repreneurs permet d'établir l'association entre le modèle du repreneur non contraint et la catégorie des entreprises dont l'effectif est en hausse (cf. figure 4).

L'absence de contraintes au démarrage pour les repreneurs concourt donc au développement de l'emploi dans l'entreprise ce qui n'est pas nécessairement le cas pour les créateurs. On note une similitude des modalités des variables illustratives sur les associations présentées en annexe 6 . 


\section{III- LES ELEMENTS D'UNE CARACTERISATION REGIONALE DES NOUVEAUX ENTREPRENEURS}

Après une présentation de l'intensité entrepreneuriale et de la survie à l'échelle régionale, nous recherchons comment les différents profils de créateurs et de repreneurs, ainsi que leurs associations avec l'évolution des effectifs salariés, s'intègrent dans l'espace régional français. Nous étudions également si l'opposition des régions relatives au profil des créateurs reproduit celle relative au profil des repreneurs et enfin si l'opposition des régions fondée sur les profils des nouveaux entrepreneurs diffère selon le devenir de l'entreprise créée (évolution de l'effectif salarié).

\subsection{La répartition des créateurs et des repreneurs dans les régions françaises :}

L'intensité entrepreneuriale (mesurée à partir des ratios nombre de créateurs et/ou nombre de repreneurs pour 10000 habitants) est plus ou moins importante dans les différentes régions françaises (cf Tableau 3). Il apparaît que la région capitale et les régions du Sud de la France (Languedoc-Roussillon, Provence-Alpes Côte d'Azur, Aquitaine, Midi-Pyrénées) ainsi que la région Rhône-Alpes ont la plus forte intensité entrepreneuriale. La disparité des régions françaises en terme d'intensité entrepreneuriale est plus marquée dans la population des créateurs que pour celle des repreneurs et la forte intensité entrepreneuriale de ces régions s'explique principalement par un nombre de créateurs pour 10000 habitants élevé et par un poids relativement important en termes d'événement entrepreneurial des créations par rapport aux reprises. C'est particulièrement le cas pour l'Île-de-France qui se distingue par une très forte intensité entrepreneuriale sur la seule population des créateurs ex-nihilo. On remarque que les régions de vieille industrialisation du Nord et de l'Est de la France (Franche-Comté, Champagne-Ardenne, Picardie, Lorraine, Nord-Pasde-Calais) ainsi que le Limousin et le Centre sont des régions à faible intensité entrepreneuriale.

Une très forte intensité entrepreneuriale globale (cf. colonne 1, tableau 3) se traduit en général par un plus fort risque d'échec entrepreneurial à 4 ans $^{18}$. Les écarts entre les régions ne sont pas liés cependant qu'à des effets de structure (importance des créateurs dans la population totale des nouveaux entrepreneurs) mais sont également le reflet de spécificités régionales (Bonneau et Thirion, 1997). C'est pourquoi les régions telles que Midi-Pyrénées et Alsace bien que comptabilisant une part importante de créateurs ex-nihilo dans l'événement entrepreneurial enregistrent des taux de survie relativement élevés.

\footnotetext{
${ }^{18}$ Ce résultat s'explique par la moindre survie des créateurs ex-nihilo par rapport aux repreneurs. Abdesselam ,Bonnet, Le Pape (1999).
} 


\begin{tabular}{|c|c|c|c|c|c|}
\hline & \begin{tabular}{|c|} 
Nombre de créateurs \\
et de repreneurs pour \\
10000 habitants
\end{tabular} & $\begin{array}{c}\text { Nombre de créateurs pour } \\
10000 \text { habitants }\end{array}$ & $\begin{array}{l}\text { Nombre de repreneurs } \\
\text { pour } 10000 \text { habitants }\end{array}$ & \begin{tabular}{|l} 
Poids de la région en \\
termes de repreneurs sur \\
poids de la région en \\
termes de créateurs
\end{tabular} & $\begin{array}{c}\text { Répartition des } \\
\text { créateurs et des } \\
\text { repreneurs }\end{array}$ \\
\hline Île-de-France & $40,58 * *_{-}$ & $36,44 * *_{-}$ & $4,13 * *_{-}$ & $0,42^{\circ}$ & $24,48 \%$ \\
\hline Languedoc-Roussillon & $37,97 * *_{-}$ & $30,04 * *$ & $7,93^{* *_{-}}$ & 1,03 & $4,94 \%$ \\
\hline Prov-Alpes-Côte d'Azur & $37,04 * *_{-}$ & $29,47 * *$ & $7,57 *_{-}$ & 0,94 & $8,68 \%$ \\
\hline Midi-Pyrénées & $33,02 * *+$ & 23,73 & $9,28 *+$ & 1,39 & $5,07 \%$ \\
\hline Aquitaine & $32,55 *+$ & 25,3 & 7,24 & 1,09 & $5,74 \%$ \\
\hline Rhône-Alpes & 32,30 & 25,42 & 6,88 & 0,97 & $10,49 \%$ \\
\hline Alsace & $25,16 * *+$ & $19,46 * *+$ & 5,7 & 1,00 & $2,70 \%$ \\
\hline Auvergne & 23,71 & 15,91 & $7,8 * *+$ & 1,74 & $1,92 \%$ \\
\hline Poitou-Charentes & $23,09 * *+$ & $17,05 * *+$ & 6,04 & 1,27 & $2,43 \%$ \\
\hline Pays De La Loire & $22,58 * *+$ & 16,28 & $6,3 * *+$ & 1,35 & $4,65 \%$ \\
\hline Bourgogne & $22,55 *+$ & 14,83 & 7,72 & 1,81 & $2,38 \%$ \\
\hline Bretagne & $22,47 * *+$ & $15,07 * *+$ & 7,39 & 1,73 & $4,10 \%$ \\
\hline Franche Comté & $22,37 * *+$ & $15,57 * *+$ & 6,8 & 1,62 & $1,51 \%$ \\
\hline Centre & 21,78 & 16,15 & $5,63 *+$ & 1,20 & $3,55 \%$ \\
\hline Basse-Normandie & 21,46 & $14,38 * *+$ & $7,08 * *+$ & 1,87 & $2,00 \%$ \\
\hline Haute-Normandie & 20,85 & 14,88 & 5,96 & 1,40 & $2,20 \%$ \\
\hline Picardie & 20,68 & 15,95 & 4,73 & 1,07 & $2,41 \%$ \\
\hline Limousin & $20,66 * *+$ & 13,4 & $7,26 * *+$ & 1,92 & $0,91 \%$ \\
\hline Lorraine & 20,28 & 15,03 & 5,24 & 1,22 & $2,99 \%$ \\
\hline Champagne-Ardenne & 20,17 & 15 & 5,16 & 1,21 & $1,78 \%$ \\
\hline Nord-Pas-De-Calais & 17,63 & 12,6 & $5,03^{* * *_{-}}$ & 1,43 & $4,55 \%$ \\
\hline Corse & 41,95 & 32,37 & 9,58 & 0,98 & $0,53 \%$ \\
\hline Total & 29,05 & 22,86 & 6,19 & 1,00 & $100 \%$ \\
\hline
\end{tabular}

Source : élaboration propre à partir de l'enquête Sine 1994 et des chiffres de l'Insee pour la population.

Situation de l'entrepreneur en 1997 : en activité ou échec entrepreneurial.

** Significativité à moins de $1 \%, *[1 \%-5 \%],+$ entrepreneurs en activité, - entrepreneurs en échec entrepreneurial.

'La valeur 0,42 pour la région Île de France correspond au rapport des $11,77 \%$ de repreneurs sur les $28,27 \%$ de créateurs que cette région comptabilise à l'échelle nationale. Donc si une région obtient la valeur 1 cela signifie qu'en moyenne elle n'a pas une spécificité marquée sur le type d'évènement entrepreneurial. Pour une information sur la part des créateurs dans le total des nouveaux entrepreneurs (cf. annexe 7).

\section{Tableau 3 : Intensité entrepreneuriale, distribution spatiale des créateurs et des repreneurs en 1994 et survie des nouvelles entreprises}

Le ratio poids de la région en termes de repreneurs rapporté au poids de la région en termes de créateurs est égal à 0,42 en Île-de-France : il montre ainsi la prépondérance des créateurs purs dans l'acte entrepreneurial au sein de cette région ${ }^{19}$. Du fait du poids important de la région capitale en termes d'évènement entrepreneurial $(24,48 \%$ du total français), un grand nombre de régions françaises se caractérisent par une valeur de ce ratio supérieure à l'unité. Le Limousin, avec un ratio de 1,92 apparaît comme la région dans laquelle le renouvellement du tissus productif se fait le plus par reprise.

Entre 1994 et 2002 l'intensité entrepreneuriale s'est en moyenne accrûe que ce soit sur la population des créateurs ou celle des repreneurs (cf. tableau 4). Les régions Provence-Alpes Côte

${ }^{19}$ Celle-ci fortement urbanisée offre de nombreuses opportunités de créations de nouvelles entreprises et ce particulièrement dans le secteur des services aux entreprises -pour ce type d'activité, la région offre plutôt de meilleures chances de survie à 4 ans (Sine 1994)-. 
d'Azur et Languedoc-Roussillon sont devenues les régions à plus forte intensité entrepreneuriale et, à l'exception de l'Île-de-France, elles dépassent de plus de 10 points les autres régions françaises. Dans le total des créateurs et des repreneurs, seules les régions île de France, Languedoc-Roussillon, Provence-Alpes Côte d'Azur et Rhône-Alpes voient leur poids augmenter.

\begin{tabular}{|c|c|c|c|c|c|}
\hline & \begin{tabular}{|c|} 
Nombre de créateurs \\
et de repreneurs pour \\
10000 habitants
\end{tabular} & $\begin{array}{c}\text { Nombre de } \\
\text { créateurs pour } \\
10000 \\
\text { habitants }\end{array}$ & \begin{tabular}{|c|} 
Nombre de \\
repreneurs pour 10 \\
000 habitants
\end{tabular} & \begin{tabular}{|c|} 
Poids de la région en \\
termes de repreneurs sur \\
poids de la région en \\
termes de créateurs
\end{tabular} & $\begin{array}{l}\text { Répartition des créateurs } \\
\text { et des repreneurs }\end{array}$ \\
\hline Pr. Alpes-Côte-Azur & 54,49 & 46,15 & 8,34 & 0,77 & $11,88 \%$ \\
\hline Languedoc-Roussillon & 50,81 & 42,08 & 8,74 & 0,88 & $5,64 \%$ \\
\hline Ile-de-France & 48,79 & 43,22 & 5,57 & 0,55 & $25,84 \%$ \\
\hline Rhône-Alpes & 39,42 & 31,47 & 7,95 & 1,07 & $10,76 \%$ \\
\hline Midi-Pyrénées & 37,29 & 29,94 & 7,36 & 1,04 & $4,60 \%$ \\
\hline Aquitaine & 36,9 & 29,4 & 7,5 & 1,08 & $5,19 \%$ \\
\hline Bretagne & 28,45 & 20,35 & 8,1 & 1,68 & $4,00 \%$ \\
\hline Alsace & 28,04 & 22,68 & 5,36 & 1,00 & $2,35 \%$ \\
\hline Pays de la Loire & 27,81 & 21,07 & 6,73 & 1,35 & $4,33 \%$ \\
\hline Poitou-Charentes & 26,99 & 19,18 & 7,82 & 1,73 & $2,14 \%$ \\
\hline Auvergne & 26,68 & 19,27 & 7,41 & 1,63 & $1,69 \%$ \\
\hline Basse-Normandie & 26,11 & 18,23 & 7,89 & 1,83 & $1,80 \%$ \\
\hline Franche-comté & 25,81 & 18,56 & 7,25 & 1,65 & $1,39 \%$ \\
\hline Bourgogne & 25,13 & 18,22 & 6,91 & 1,61 & $1,96 \%$ \\
\hline Limousin & 24,49 & 17,85 & 6,64 & 1,58 & $0,84 \%$ \\
\hline Lorraine & 24,2 & 18,58 & 5,62 & 1,28 & $2,70 \%$ \\
\hline Centre & 23,85 & 17,73 & 6,12 & 1,46 & $2,81 \%$ \\
\hline Champagne-Ardenne & 22,84 & 16,96 & 5,89 & 1,47 & $1,48 \%$ \\
\hline Haute-Normandie & 21,54 & 15,87 & 5,67 & 1,51 & $1,85 \%$ \\
\hline Picardie & 20,96 & 15,9 & 5,07 & 1,34 & $1,88 \%$ \\
\hline Nord-pas-de-calais & 20,81 & 15,91 & 4,9 & 1,30 & $4,02 \%$ \\
\hline Corse & 64,95 & 50,81 & 14,14 & 1,18 & $0,82 \%$ \\
\hline Total & 35,33 & 28,58 & 6,75 & 1,00 & $100 \%$ \\
\hline
\end{tabular}

Elaboration propre à partir des données APCE 2002.

\section{Tableau 4 : Intensité entrepreneuriale et distribution spatiale des créateurs et des repreneurs en 2002.}




\subsection{La caractérisation des régions selon les profils des créateurs et selon les profils des repreneurs :}

Une typologie des régions françaises a été réalisée selon les quatre profils de créateurs et les quatre profils de repreneurs (cf. tableau 2). Les résultats nous ont amené à retenir quatre classes homogènes de régions selon les profils des créateurs et trois classes homogènes selon les profils des repreneurs (cf cartes 1 et 2). Cette analyse nous permet de mettre en évidence quels sont les profils des créateurs et des repreneurs qui différencient au mieux les régions françaises.

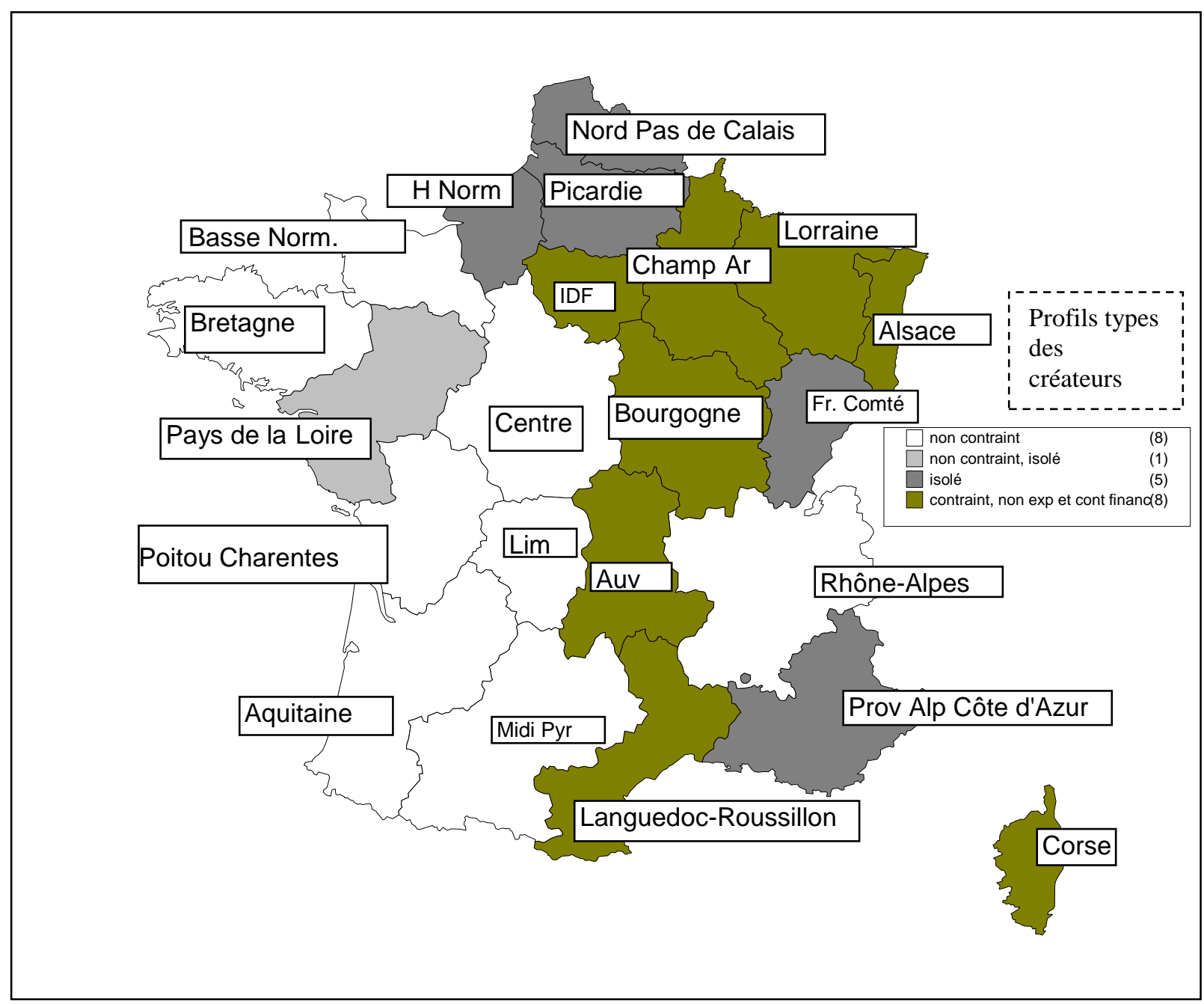

Résultats $\mathrm{AFC}+\mathrm{CHA}$

Carte 1 : positionnement des régions françaises relativement aux profils des créateurs 


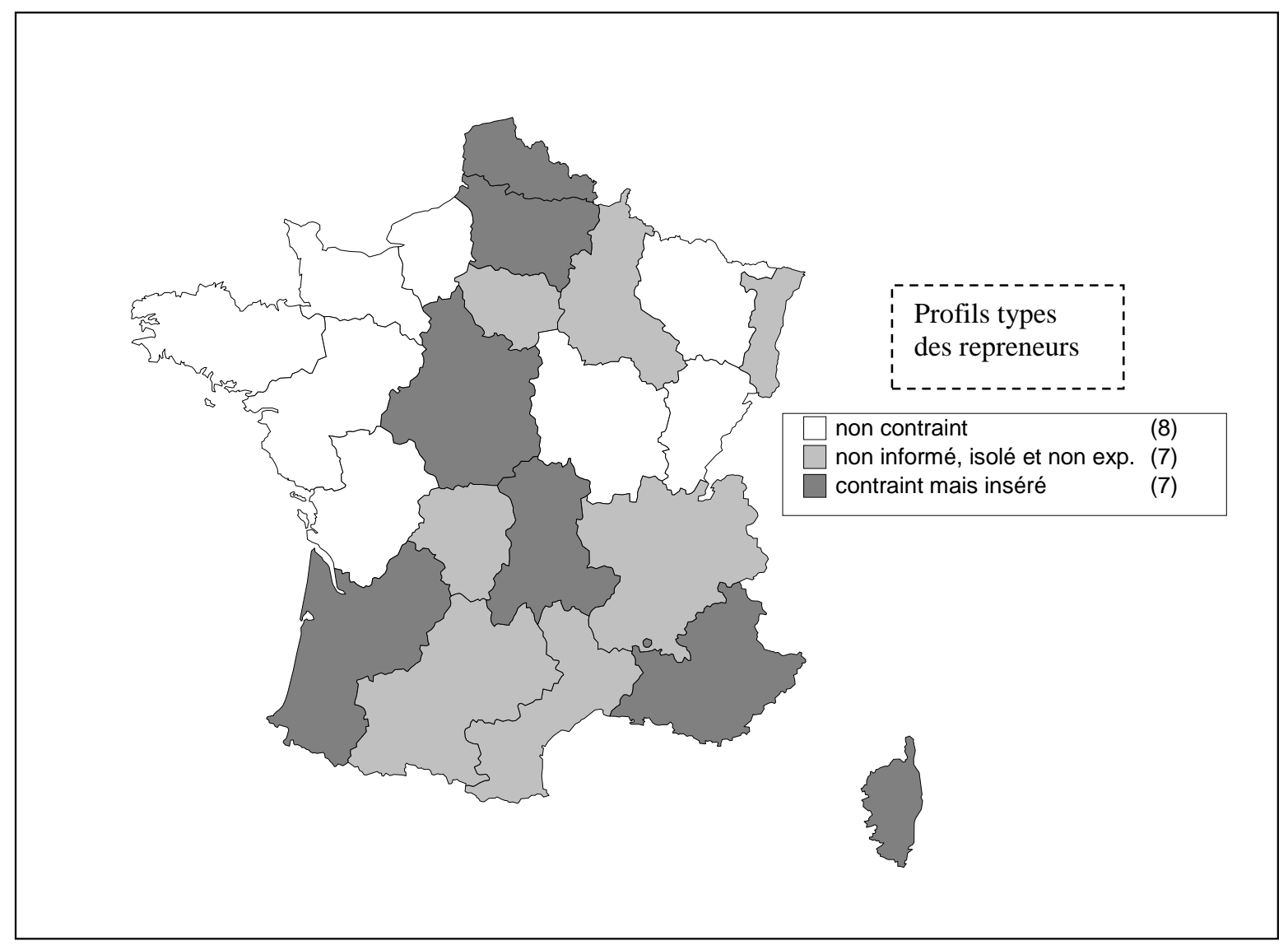

\section{Résultats $\mathrm{AFC}+\mathrm{CHA}$}

\section{Carte 2 : positionnement des régions françaises relativement aux profils des repreneurs}

Globalement mais à l'exception notable de la région Rhône-Alpes, une opposition entre les régions Ouest et Est de la France apparaît quant à l'intensité des contraintes subies par les créateurs purs : les régions de l'Ouest relèvent globalement d'un profil de créateur moins contraint. En ce qui concerne les repreneurs, l'ensemble de régions du Sud de la France relèvent d'un modèle contraint.

Certaines régions françaises ressortent comme étant caractéristiques du seul profil du créateur non contraint ou de celui du repreneur non contraint ${ }^{20}$ alors qu'au sein d'autres régions deux profils apparaissent.

On observe que le modèle "non contraint" à la fois sur les populations de créateurs et de repreneurs se retrouve uniquement dans trois régions françaises : Basse-Normandie, Bretagne et Poitou-Charentes. Pour les Pays de la Loire, le profil caractéristique du créateur est non contraint ou isolé. Il apparaît donc que ces régions de "culture entrepreneuriale" (c'est-à-dire qui présentent un assez faible taux de chômage et des créations relativement importantes : Pays de la Loire, Bretagne, Poitou-Charentes), sont aussi celles ou les nouveaux entrepreneurs subissent a priori le moins de contraintes au démarrage. A l'opposé, les régions Bourgogne et Lorraine qui ressortent de la catégorie des repreneurs non contraints se retrouvent dans l'ensemble des créateurs contraints

\footnotetext{
${ }^{20}$ Les repreneurs des régions correspondant au profil du "repreneur non contraint" se caractérisent par un âge relativement jeune, une motivation à la reprise liée au goût d'entreprendre et la nationalité française. Ils reprennent plutôt dans les secteurs de l'industrie agroalimentaire et des hôtels-cafés-restaurants.
} 
ou non expérimentés et contraints financièrement. Autrement dit, au niveau régional, une certaine hétérogénéité ressort quant à l'intensité des contraintes selon la modalité de l'acte entrepreneurial.

\subsection{La caractérisation des régions selon la création d'emplois par les créateurs et selon}

\section{la création d'emplois par les repreneurs :}

Une typologie des régions françaises a été réalisée selon les quatre classes caractérisant la création d'emplois (cf. tableau 2). Les résultats nous ont amené à retenir quatre classes homogènes de régions selon l'évolution de l'emploi par les créateurs et quatre classes homogènes selon l'évolution de l'emploi par les repreneurs (cf carte 3 et 4).

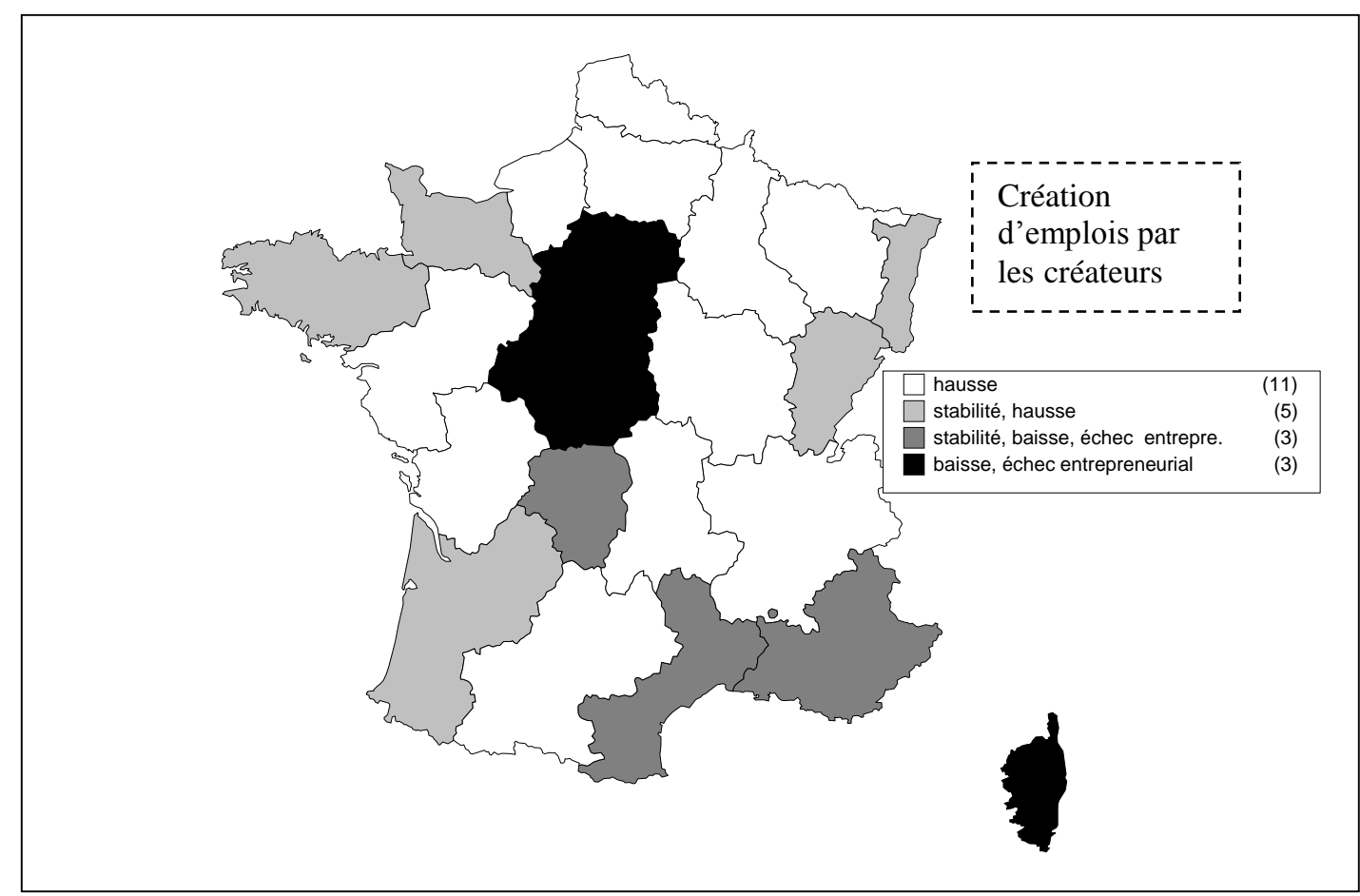

Résultats $\mathrm{AFC}+\mathrm{CHA}$

\section{Carte 3 : Positionnement des régions françaises relativement à la création d'emplois par les créateurs}

Deux groupes de régions françaises s'opposent selon la création d'emplois par les créateurs. Le premier regroupe les deux classes pour lesquelles on observe une évolution défavorable de la création d'emplois (échec entrepreneurial, baisse de l'effectif salarié) et comprend l'Île de France, Provence-Alpes-Côte-d'Azur et Languedoc-Roussillon ${ }^{21}$. Ces trois régions sont caractérisées par une forte intensité entrepreneuriale et par un poids important des créateurs dans l'ensemble des créateurs et des repreneurs (cf. supra). Le poids de la région Île de France dans l'ensemble des créateurs associé à la faible survie des créations ex-nihilo dans cette région tire vers le haut les autres régions françaises. Aussi un second groupe comprend un

${ }^{21}$ mais aussi Centre, Limousin et Corse. 
ensemble plus large de régions dans lesquelles les créateurs d'entreprises enregistrent une stabilité voire une hausse de leurs effectifs salariés ${ }^{22}$ (16 des 22 régions françaises).

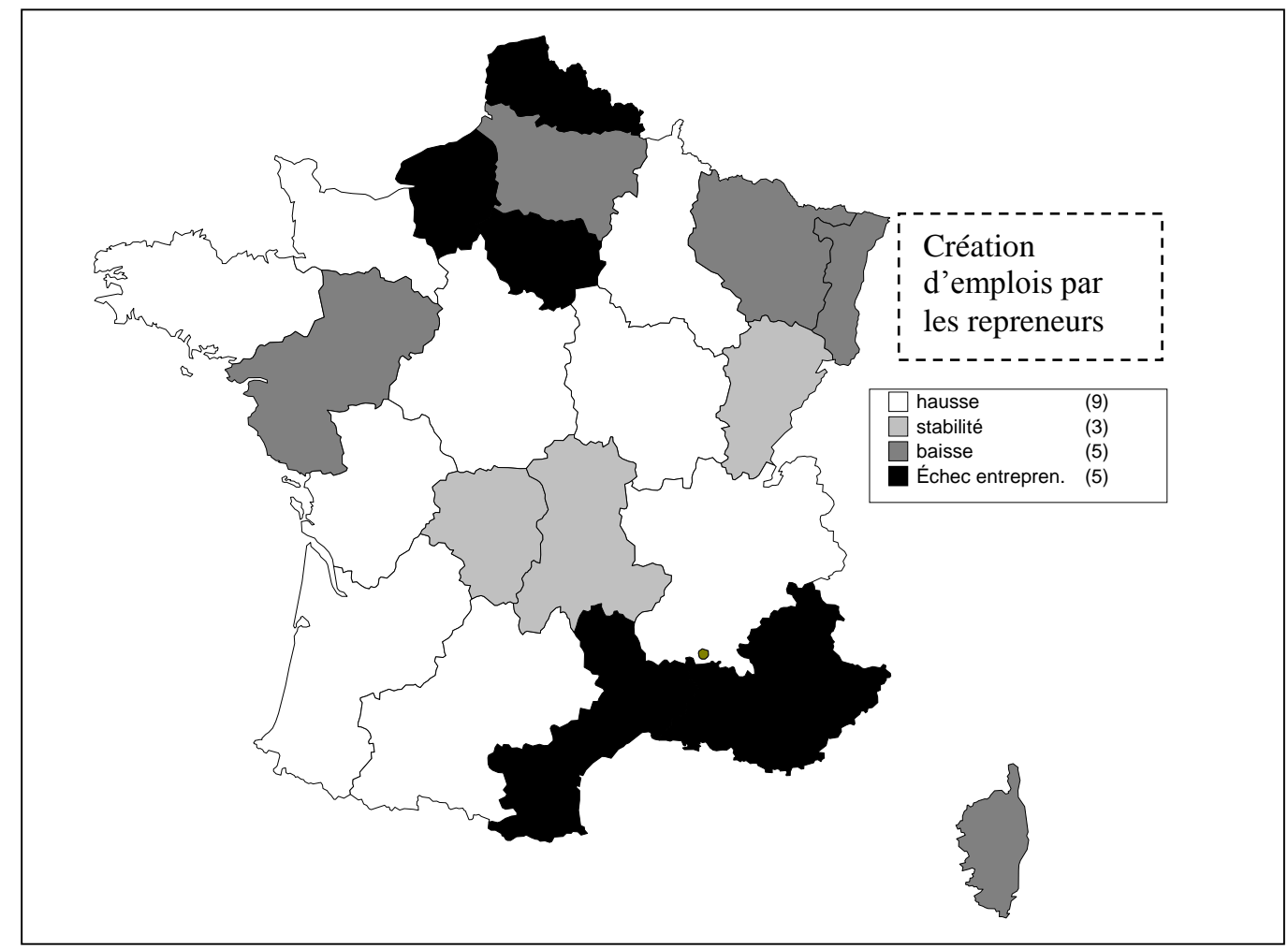

Résultats $\mathrm{AFC}+\mathrm{CHA}$

\section{Carte 4: Positionnement des régions françaises relativement à la création d'emplois par les repreneurs}

En ce qui concerne les repreneurs, l'île de France, Provence-Alpes-Côte-d'Azur et Languedoc-Roussillon mais aussi la Haute-Normandie et le Nord-Pas-de-Calais relèvent de la catégorie de l'échec entrepreneurial. Dans la classe des baisses d'effectifs salariés, nous avons la Corse, la Picardie, les Pays de la Loire, la Lorraine et l'Alsace. Les régions stables sont la FrancheComté, le Limousin et l'Auvergne. Pour cette dernière région, la part des reprises est importante.

On remarque également un profil différencié des régions selon l'évènement entrepreneurial. Ainsi, le Nord-Pas-de-Calais, la Haute-Normandie, la Picardie, la Lorraine, l'Alsace et les Pays de la Loire sont des régions pour lesquelles les créateurs enregistrent une évolution assez favorable de leurs effectifs salariés ce qui n'est pas le cas pour les repreneurs. On observe l'inverse pour le Centre et le Limousin.

En revanche, l'Île de France, Provence-Alpes-Côte-d'Azur, Languedoc-Roussillon et Corse ont le même profil quelque soit l'événement entrepreneurial. Dans ces régions les nouveaux entrepreneurs subissent soit un échec entrepreneurial soit une baisse d'effectifs salariés. Inversement certaines régions se caractérisent par un environnement propice à la création d'emplois aussi bien pour les créateurs que pour les repreneurs (Bretagne, Basse-Normandie, Aquitaine, Midi Pyrénées, Poitou-Charentes, Bourgogne, Franche-Comté, Lorraine et Rhône-

\footnotetext{
${ }^{22}$ Les variables illustratives montrent les éléments d'une différenciation régionale des profils des créateurs sur ces deux groupes de régions. Au sein du groupe de régions qui sont caractérisées par une stabilité ou une croissance de leurs effectifs salariés par les créateurs ex-nihilo (et à la différence de l'autre groupe de régions), ces créateurs sont plutôt jeunes, de sexe masculin et de nationalité française et créent dans le secteur de l'industrie.
} 
Alpes). Enfin certaines régions frontalières dont une partie de l'activité est tirée par les relations transfrontalières et une forte culture entrepreneuriale (Franche-Comté et Rhône-Alpes mais aussi l'Alsace pour les créateurs), se caractérisent par une hausse ou une stabilité de leurs effectifs salariés.

\subsection{La caractérisation des régions selon les contraintes et la création d'emplois :}

Nous avons ensuite croisé nos modèles de créateurs et de repreneurs avec l'évolution des effectifs salariés. Nous obtenons 16 classes par événement entrepreneurial (par exemple non contraint/baisse, Non contraint/hausse etc...) ce qui nous permet d'apprécier comment l'espace déforme la performance de l'entreprise relativement aux contraintes que l'entrepreneur subit.

La segmentation globale sur les créateurs est plus claire que sur les repreneurs. Peut-on en déduire pour autant que les contraintes sont moins pertinentes pour la reprise?

Pour les créateurs, les 5 classes homogènes d'associations relevées sont les suivantes :

Classe 1 : elle comprend l'Île de France, la Lorraine et la Corse. On a une domination des modèles de créateurs contraints sans différenciation de l'évolution future de l'entreprise créée. En revanche pour les 2 autres types de créateurs contraints (isolé et non expérimenté, contrainte financière) l'évolution de l'entreprise est défavorable. Cette classe est caractérisée par le fait qu'elle regroupe des créateurs d'origine étrangère (hors union européenne et union européenne) et elle est aussi composée de plusieurs sous populations, des cadres, actifs de niveau Bac plus deux et plus mais aussi des inactifs sans diplôme. La motivation est alors la nouvelle idée mais aussi l'exemple de l'entourage.

Toutes les autres classes de régions font ressortir des créateurs d'origine française.

Classe 2 : la présence de contraintes ne semble pas être un handicap dans ce groupe de régions qui comprend : la Champagne-Ardenne, la Picardie, la Haute-Normandie, la Bourgogne, le Nord-Pas-de-Calais, 1'Alsace, Midi Pyrénées et l'Auvergne. On trouve en effet les classes isolé/hausse, isolé/stable, contraint/hausse. La population des créateurs est composée de jeunes ouvriers de formation technique ou sans diplôme, souvent au chômage et dont la motivation est de retrouver un emploi.

Classe 3: dans cette classe, les modèles non contraints sont associés à une évolution défavorable des entreprises. Elle est composée des régions Provence-Alpes-Côte-d'Azur et Languedoc-Roussillon. Cette classe comprend de façon significative des femmes inactives ou au chômage depuis plus d'un an.

Classe 4: elle représente la domination du modèle non contraint avec cependant des évolutions diverses en ce qui concerne les effectifs salariés. Elle regroupe le Centre, la BasseNormandie et Rhône-Alpes. Le créateur est généralement un ouvrier dont le niveau de formation est le CAP/BEP/BEPC.

Classe 5 : il n'y a pas de détermination en ce qui concerne l'évolution des effectifs salariés pour les créateurs non contraints mais une évolution assez favorable des créateurs contraints. Cette classe regroupe la Franche-Comté, les Pays de la Loire, la Bretagne, le Poitou-Charentes, l'Aquitaine et le Limousin. Le créateur est employé ou de profession intermédiaire, il possède un bac général et technique ou un CAP/BEP/BEPC. La motivation de la création est l'opportunité. 
Pour les repreneurs, 5 classes homogènes d'associations sont aussi choisies. Cependant, globalement, il n'y a pas d'associations du modèle non contraint avec une évolution favorable sur les effectifs salariés.

Classe 1: On retrouve l'île de France, seule, caractérisée par des repreneurs contraints d'origine étrangère et qui enregistrent une évolution défavorable de leurs entreprises.

Classe 2 : elle regroupe Provence-Alpes-Côte-d'Azur et la Corse. C'est la dominance du contraint/inséré pour lequel cependant l'évolution n'est pas déterminée. On retrouve aussi non informé/baisse et Isolé non expérimenté/mortes.

Pour les autres classes, il plus difficile d'interpréter les couples contraintes performances de l'entreprise et on a du mal à dégager clairement une caractérisation régionale.

\section{Conclusion}

Les contraintes liées à l'acte entrepreneurial et la création d'emplois par les nouveaux entrepreneurs entretiennent des relations complexes dans l'espace régional français. Cependant quelques points marquants ressortent.

La région capitale apparaît comme spécifique avec une intensité entrepreneuriale très forte ${ }^{23}$, une part des créateurs très importante dans le total des nouveaux entrepreneurs (par ailleurs le plus souvent confrontés à un échec entrepreneurial). Cette région se caractérise étonnamment par des créateurs et des repreneurs contraints. Une explication possible réside dans le fait que cette région accueille de façon significative des entrepreneurs étrangers. Cette région contribuerait à travers l'entrepreneuriat à participer à une certaine intégration sociale.

Les régions du Languedoc-Roussillon et de Provence-Alpes Côte d'Azur sont les régions les plus attractives dans l'espace Français depuis une vingtaine d'années maintenant. Il en résulte un fort chômage qualifié de chômage de croissance mais aussi une forte intensité entrepreneuriale ${ }^{24}$. Les performances des nouveaux entrepreneurs sont mauvaises et nous avons affaire à des individus contraints.

Les régions de vieille industrialisation en reconversion et à fort taux de chômage (Nord-Pasde-Calais, Champagne-Ardennes, Picardie et dans une moindre mesure la Lorraine) sont des régions de faible intensité entrepreneuriale et à entrepreneurs contraints. Cependant leurs performances sont inégales en ce qui concerne la création d'emplois selon la nature de l'événement entrepreneurial. Ces quatre régions enregistrent une croissance de l'emploi véhiculé par les créateurs alors que seule la Lorraine présente la même caractéristique pour les repreneurs.

Au niveau national, l'échec entrepreneurial est clairement relié à la présence de contraintes au démarrage que ce soit pour les créateurs ou pour les repreneurs. Une politique publique visant à

\footnotetext{
${ }^{23}$ Différents travaux (Ashcroft B., Love J.H. et E. Malloy, 1991, Keeble D. et S. Walkers, 1993, Guesnier B., 1994) montrent que la création d'entreprise est favorisée par l'urbanité en particulier par un effet demande qui se manifeste dans les grandes agglomérations surtout dans les secteurs des services aux ménages et aux entreprises et dans une moindre mesure dans les secteurs du commerce et de l'artisanat.

${ }^{24}$ A titre illustratif, pour la région Provence-Alpes-Côte d'Azur, les migrations de personnes âgées sont souvent avancées pour expliquer les créations d'entreprises dans le secteur des services aux ménages et du commerce. On constate aussi la migration de familles d'actifs vers les régions Languedoc-Roussillon et Midi-Pyrénées qui entraîne la perte d'emploi pour un des membres de la cellule familiale et qui suscite en retour la création de nouvelles activités (Thireau, 1993).
} 
atténuer l'intensité de ces contraintes devrait contribuer à une amélioration de la pérennité des nouvelles entreprises. Cette politique aurait également une efficacité en création d'emplois plus marquée sur la population des repreneurs relativement à celle des créateurs. Ce constat reflète sans doute un modèle de croissance plus difficile à maîtriser pour les créateurs purs compte tenu d'une incertitude plus grande à laquelle ils sont confrontés (relations de clientèle à établir, conquête d'un nouveau marché, introduction d'un nouveau produit etc...). A l'échelle spatiale, une des pistes de recherche serait d'examiner si il existe des espaces territoriaux dans lesquels une politique d'aide et d'accompagnement qui atténue les contraintes au démarrage est véritablement efficace : elle devrait alors attirer vers l'acte entrepreneurial préférentiellement les profils individuels disposant d'un «capital humain entrepreneurial » (traits de caractéristiques psychologiques spécifiques qui permettent le succès d'une entreprise). 


\section{Annexe 1 : Dictionnaire des variables}

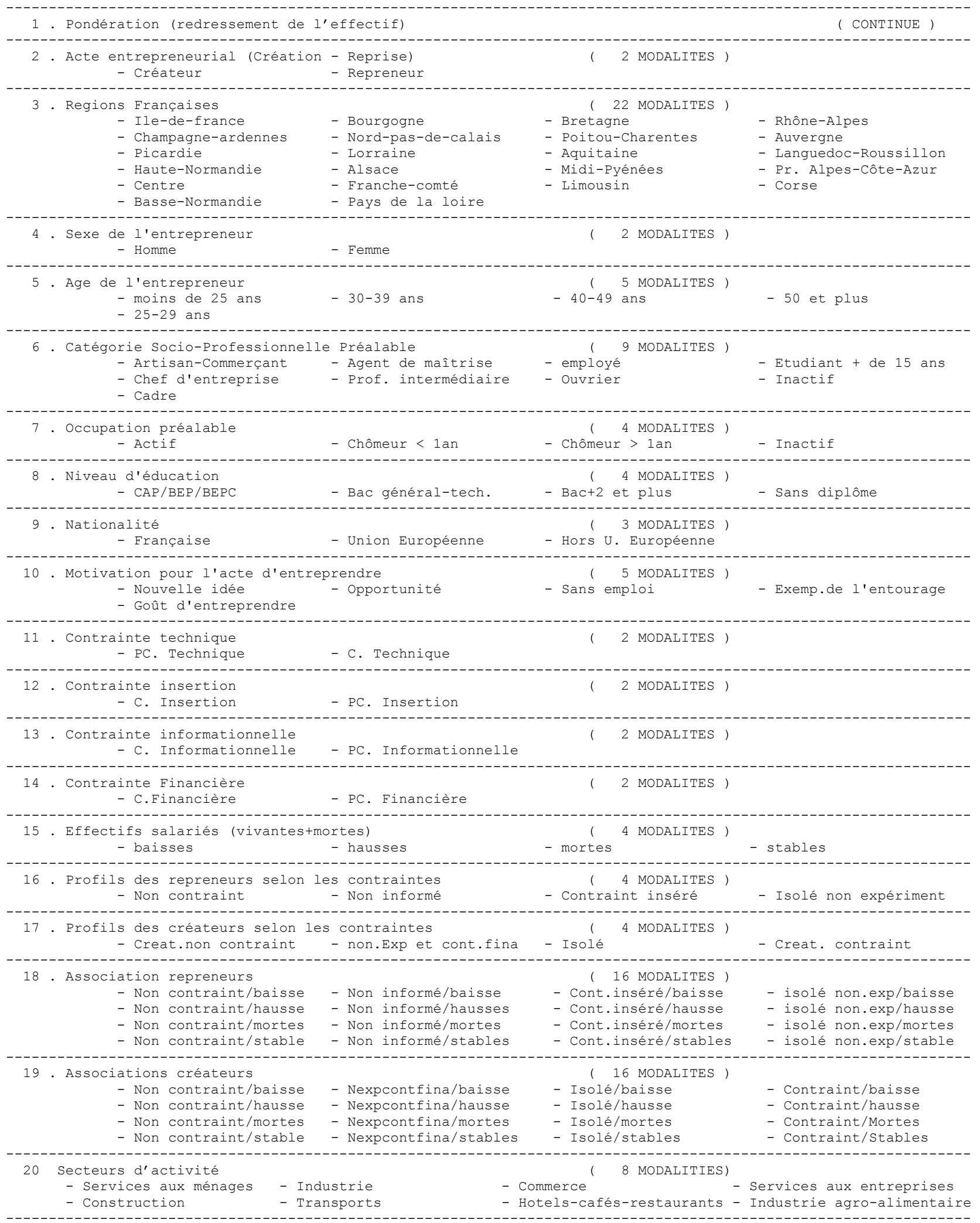


Annexe 2 : intensité entrepreneuriale selon certaines caractéristiques socioéconomiques

\begin{tabular}{|c|c|c|c|}
\hline \multicolumn{3}{|c|}{ Situation personnelle et sociale de l'individu } & \multirow{2}{*}{$\begin{array}{c}\text { Intensité } \\
\text { entrepreneuriale* } \\
+\end{array}$} \\
\hline \multirow{20}{*}{ 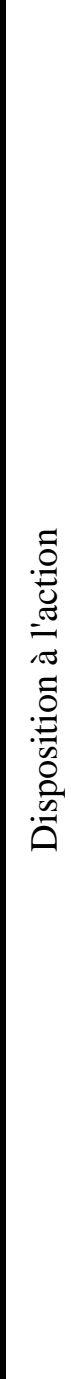 } & \multirow{2}{*}{ Sexe } & Homme & \\
\hline & & Femme & - \\
\hline & \multirow{5}{*}{ Age } & Moins de 25 ans & - \\
\hline & & $25-29$ ans & + \\
\hline & & 30-39 ans & + \\
\hline & & $40-49$ ans & + \\
\hline & & 50 ans et plus & - \\
\hline & \multirow{9}{*}{$\begin{array}{c}\text { Catégorie } \\
\text { Socioprofessionnelle }\end{array}$} & Artisan-commerçant & + \\
\hline & & Chef d'entreprise & + \\
\hline & & Cadre & + \\
\hline & & Agent de maîtrise & + \\
\hline & & Profession intermédiaire & - \\
\hline & & Employé & + \\
\hline & & Ouvrier & - \\
\hline & & Etudiant (plus de 15 ans) & \\
\hline & & Inactif & \\
\hline & \multirow{4}{*}{ Niveau d'éducation } & Sans diplôme & - \\
\hline & & Diplôme d'un niveau CAP/BEP/BEPC & + \\
\hline & & Diplôme du baccalauréat général ou technique & + \\
\hline & & Diplôme universitaire $\mathrm{Bac}+2$ et plus & + \\
\hline \multirow{7}{*}{ 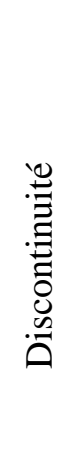 } & \multirow{4}{*}{ Activité préalable } & Population active occupée & - \\
\hline & & Chômeurs de moins d'un an & + \\
\hline & & Chômeur de plus d'un an & + \\
\hline & & Population non active & - \\
\hline & \multirow{3}{*}{ Nationalité } & Français & - \\
\hline & & Union européenne & + \\
\hline & & Hors Union européenne & + \\
\hline
\end{tabular}

Elaboration propre à partir de l'enquête Sine 1994 et les chiffres de l'Insee.

* Le signe + (-) signifie une plus forte (faible) représentation de la modalité de la variable au sein des créateurs-repreneurs d'entreprises qu'au sein de la population totale. Toutes les modalités sont significatives au seuil de $1 \%$. 


\section{Annexe 3 : l'identification des quatre types de contraintes sur les créateurs et les repreneurs}

\begin{tabular}{|c|c|c|c|}
\hline & Questions enquête SINE & Modalités des réponses & Identification des contraintes \\
\hline \multirow{4}{*}{ 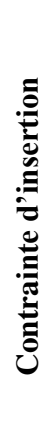 } & \multirow{4}{*}{$\begin{array}{c}\text { "Avez-vous ou avez-vous eu des } \\
\text { personnes dans votre entourage qui sont } \\
\text { ou ont été entrepreneurs?" }\end{array}$} & Non, ni de la famille ni des amis & $\Rightarrow$ Contrainte d'insertion décelée \\
\hline & & $\begin{array}{l}\text { Oui, mais uniquement des membres de la } \\
\text { famille }\end{array}$ & $\begin{array}{l}\Rightarrow \text { Contrainte d'insertion non } \\
\text { décelée }\end{array}$ \\
\hline & & Oui, mais uniquement des amis & $\begin{array}{l}\Rightarrow \text { Contrainte d'insertion non } \\
\text { décelée }\end{array}$ \\
\hline & & Oui, des membres de la famille et des amis & $\begin{array}{l}\Rightarrow \text { Contrainte d'insertion non } \\
\text { décelée }\end{array}$ \\
\hline \multirow{4}{*}{ 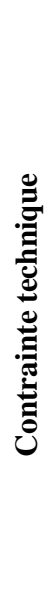 } & \multirow{4}{*}{$\begin{array}{c}\text { "Dans quel secteur avez-vous acquis des } \\
\text { compétences durant votre activité } \\
\text { précédente?" }\end{array}$} & $\begin{array}{c}\text { Dans le même secteur que celui de } \\
\text { l'entreprise créée ou reprise }\end{array}$ & $\begin{array}{l}\Rightarrow \text { Contrainte technique non } \\
\text { décelée }\end{array}$ \\
\hline & & $\begin{array}{l}\text { Dans un secteur proche de celui de } \\
\text { l'entreprise créée ou reprise }\end{array}$ & $\begin{array}{l}\Rightarrow \text { Contrainte technique non } \\
\text { décelée }\end{array}$ \\
\hline & & $\begin{array}{l}\text { Dans un secteur différent de celui de } \\
\text { l'entreprise créée ou reprise }\end{array}$ & $\Rightarrow$ Contrainte technique décelée \\
\hline & & $\begin{array}{l}\text { Dans un secteur proche de celui de } \\
\text { l'entreprise créée ou reprise mais uniquement } \\
\text { pour mon associé }\end{array}$ & $\Rightarrow$ Contrainte technique décelée \\
\hline \multirow{4}{*}{ 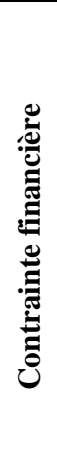 } & \multirow{2}{*}{ "Avez-vous demandé un prêt bancaire?" } & Prêt bancaire demandé et obtenu & $\begin{array}{l}\Rightarrow \text { Contrainte financière non } \\
\text { décelée }\end{array}$ \\
\hline & & Prêt bancaire demandé et non obtenu & $\Rightarrow$ Contrainte financière décelée \\
\hline & "Avez-vous obtenu ce prêt bancaire?" & $\begin{array}{l}\text { Prêt bancaire non demandé pour un montant } \\
\text { investi inférieur à } 3811 \text { Euros }\end{array}$ & $\Rightarrow$ Contrainte financière décelée \\
\hline & $\begin{array}{l}\text { "Quel est le montant investi au } \\
\text { démarrage de l'entreprise?" }\end{array}$ & $\begin{array}{c}\text { Prêt bancaire non demandé pour un montant } \\
\text { investi supérieur à } 3811 \text { Euros }\end{array}$ & $\begin{array}{l}\Rightarrow \text { Contrainte financière non } \\
\text { décelée }\end{array}$ \\
\hline \multirow{4}{*}{ 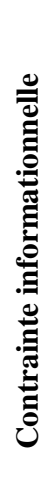 } & \multirow{4}{*}{$\begin{array}{l}\text { "Avez-vous entrepris des études } \\
\text { spécifiques, des enquêtes etc. avant de } \\
\text { créer votre entreprise?" } \\
\text { "Avez-vous pris contact avec vos clients } \\
\text { avant la création de votre entreprise?" }\end{array}$} & $\begin{array}{l}\text { Pas d'études ou d'enquêtes réalisées et pas de } \\
\text { contact avec les clients }\end{array}$ & $\begin{array}{l}\Rightarrow \text { Contrainte informationnelle } \\
\text { décelée }\end{array}$ \\
\hline & & $\begin{array}{c}\text { Pas d'études ou d'enquêtes réalisées mais } \\
\text { prise de contact avec les clients }\end{array}$ & $\begin{array}{l}\Rightarrow \text { Contrainte informationnelle } \\
\text { non décelée }\end{array}$ \\
\hline & & $\begin{array}{l}\text { Etudes ou enquêtes réalisées mais pas de } \\
\text { contact avec les clients }\end{array}$ & $\begin{array}{l}\Rightarrow \text { Contrainte informationnelle } \\
\text { non décelée }\end{array}$ \\
\hline & & $\begin{array}{l}\text { Etudes ou enquêtes réalisées et prise de } \\
\text { contact avec les clients }\end{array}$ & $\begin{array}{l}\Rightarrow \text { Contrainte informationnelle } \\
\text { non décelée }\end{array}$ \\
\hline
\end{tabular}


Annexe 4 : enchaînements méthodologiques de classifications sur facteurs principaux d'analyses factorielles des correspondances simples et multiples
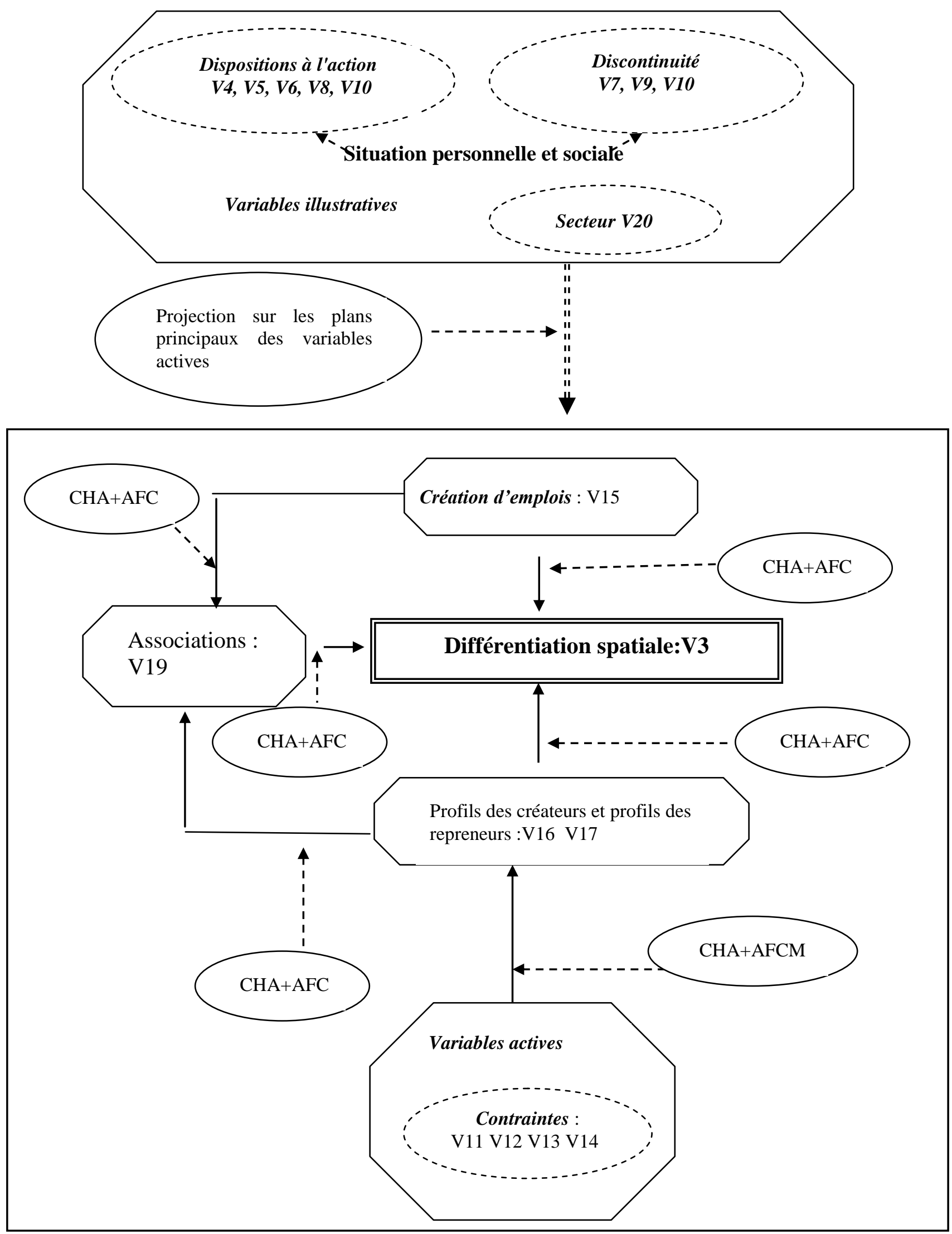
Annexe 5 : modalités des variables illustratives de deux associations entre profils de créateurs et création d'emplois
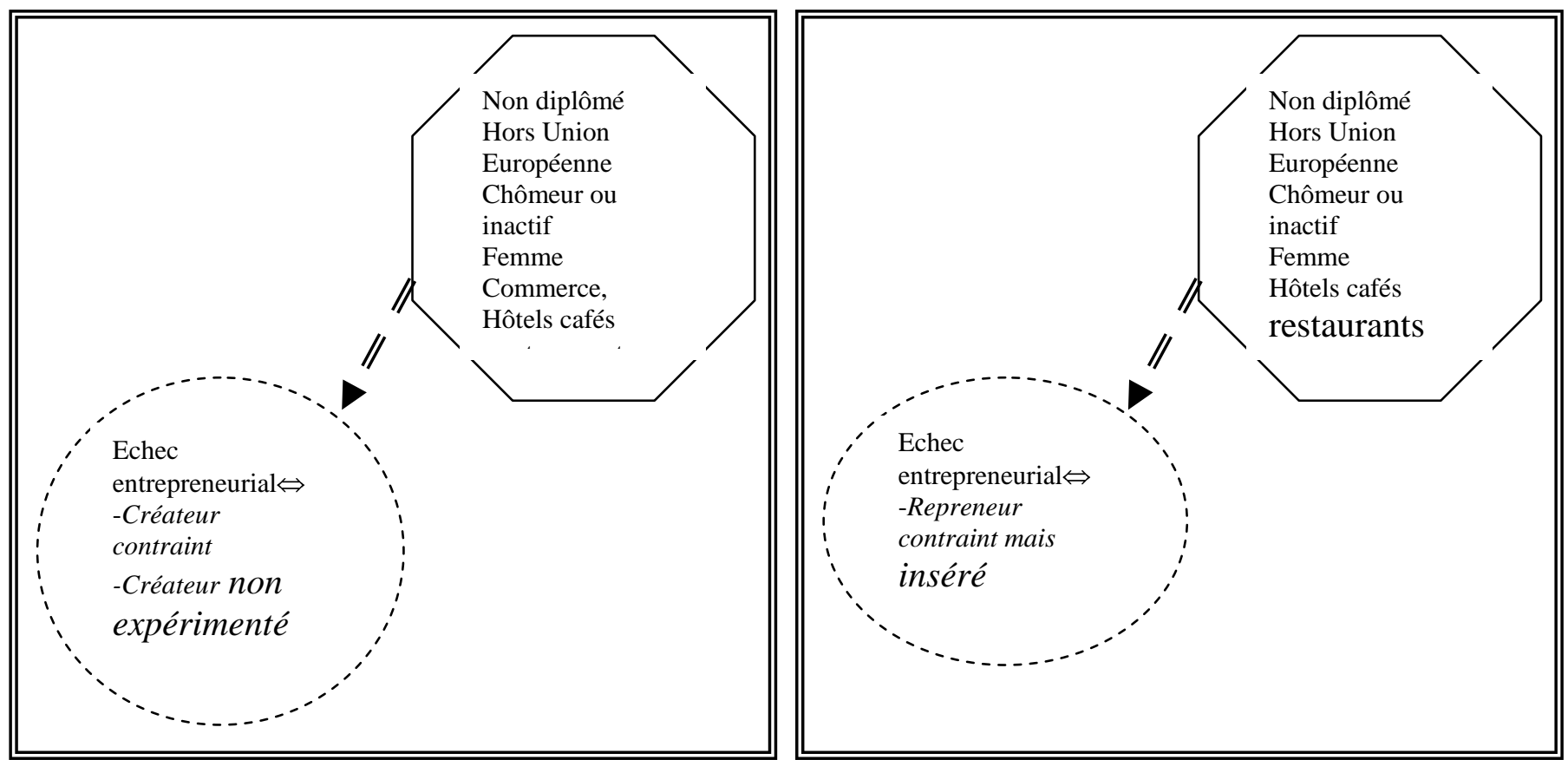

Annexe 6 : modalités des variables illustratives de deux associations entre profils de repreneurs et création d'emplois
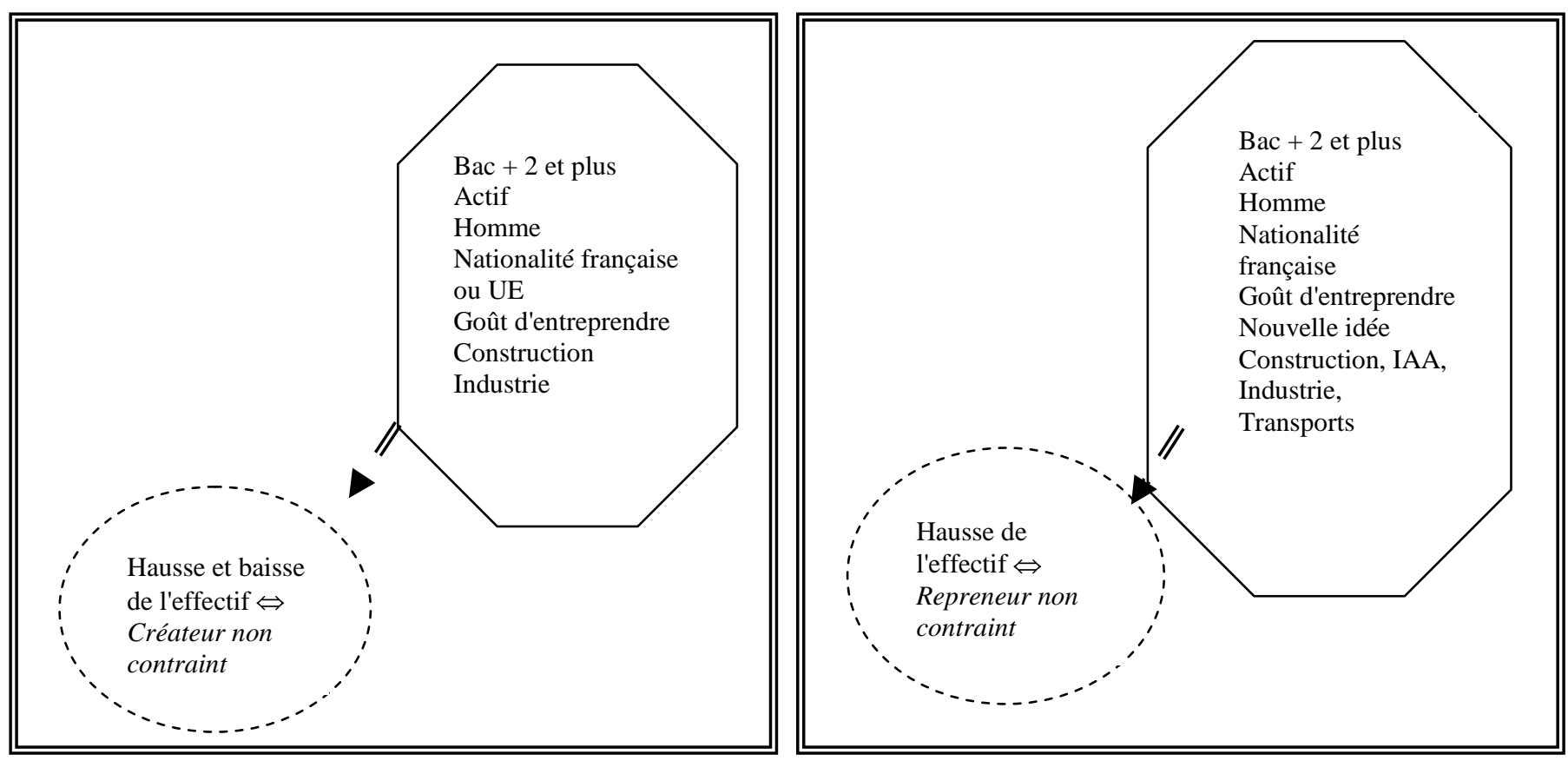
Annexe 7 : Part des créateurs ex-nihilo dans l'ensemble des créateurs et des repreneurs d'entreprises en 1994

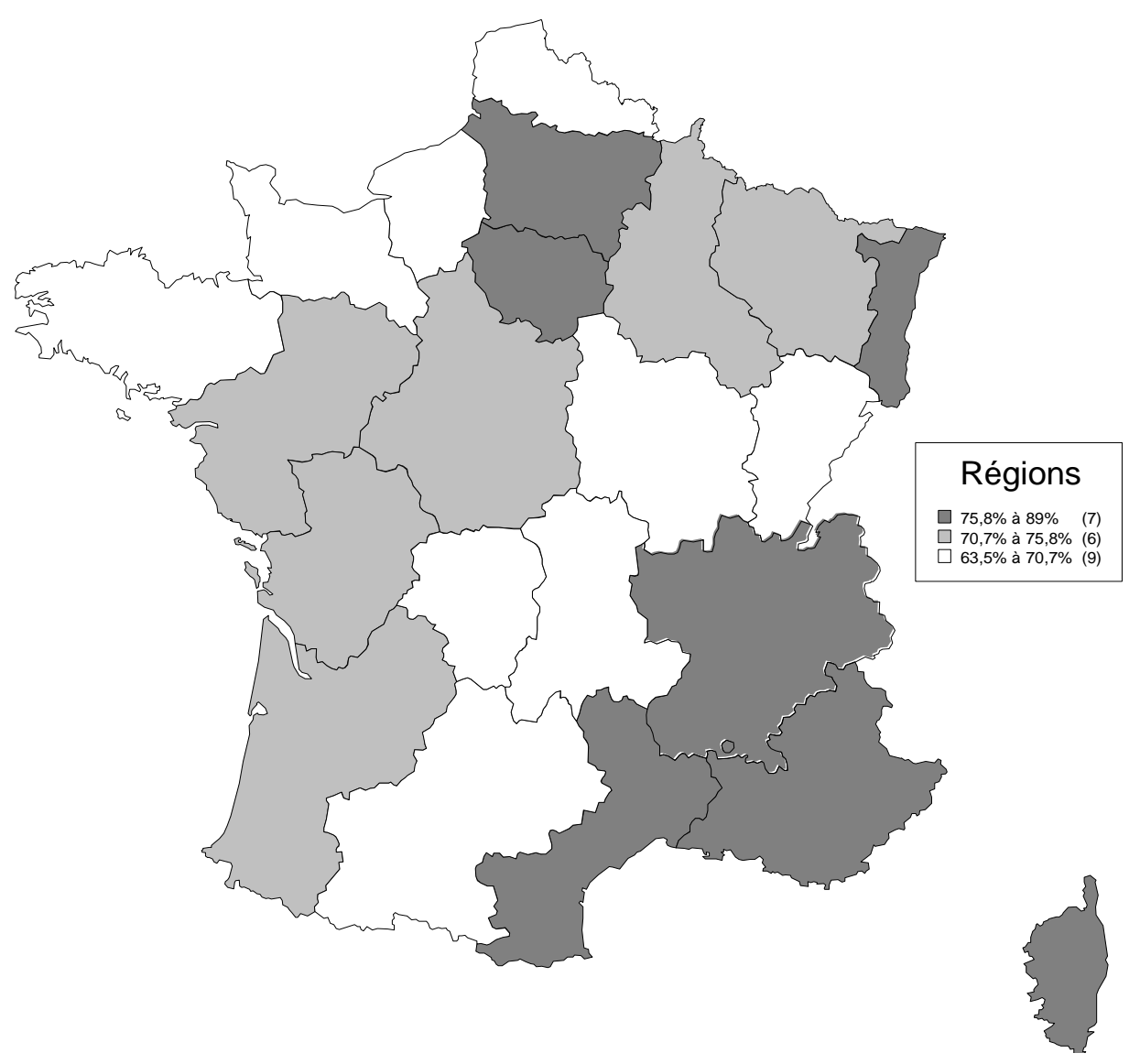




\section{Bibliographie}

ABDESSELAM R., BONNET J. et N. LE PAPE (2004), "An explanation of the life span of new french firms", à paraître dans la revue Small Business Economics.

ABDESSELAM R., BONNET J. et N. LE PAPE (1999), "Features of the entrepreneur and life span of the firm: a geographical and sector based analysis from French data". International Conference on Small Business (ICSB), 30 Septembre-02 Octobre, Rennes Saint-Malo.

ASHCROFT B., J.H. LOVE et E. MALLOY (1991), "New firms formation in the British Counties with special reference to Scotland", Regional Studies, vol.25, p. 395-409.

APCE, Agence pour la Création d'Entreprises : http://www.apce.com/index.php.

BENZECRI, J.P., 1973, L'analyse des correspondances, Dunod, Tome 2, Paris.

BONNEAU J. et B.THIRION (1997), "Création et devenir des entreprises créées de 1987 à 1995", Insee résultats, coll. système productif, $\mathrm{n}^{\circ} 125-126-127$, Avril.

BONNET J., GHARBI A. et N. LE PAPE (2003), “Debt, aggressiveness and young french firms' survival: an empirical investigation", Conference ESEM, 58th European Meeting of the Econometric Society Stockholm, August 20-24,

CARLUER F. (1998), "trois cas archétypaux de la polarisation spatio-productive : le district industriel, le milieu innovateur et la technopole. Similitudes et différences.", séminaire de l'IRER, Paris1-Sorbonne.

CIEPLY, Sylvie and Bernard PARANQUE (1998), "Le rationnement des petites entreprises sur le marché du crédit, mythe ou réalité", Banques et Marché, 33, avril.

CIEPLY S. (2001), "New Initiatives to Bridge the Financial Gap for Innovative Firms in France", Industry \& Innovation, vol. 8, $\mathrm{n}^{\circ} 2$, August, pp. 159-178.

CREPON B., DUGUET E. (2002), "Prêt bancaire, aides publiques et survie des nouvelles entreprises : une analyse économétrique à partir des méthodes d'appariement sélectif sur données d'entrepreneurs ", Eurequa, cahiers de recherches, ${ }^{\circ} 48$.

DIDAY E. (1971), “La méthode des nuées dynamiques », Revue de Statistique Appliquée, 19, №2, pp 19-34.

GLOBAL ENTREPRENEURSHIP MONITOR (2002), GEM Executive Report 2002, Rapport disponible à l'adresse http ://www.gemconsortium.org

GUISO L., SAPIENZA P. et ZINGALES L. (2002), "Does Local Financial Development Matter ?", NBER Working Paper Series, May.

GOLLAC, M. et P. LAUHLE (1987), "La transmission du statut social : l'échelle et le fossé", Economie et Statistique, 199-200, 85-93.

GUESNIER B. (1994), "Regional Variations in New Firms Formation in France", Regional Studies, vol.28, p.347-358.

HAGEN, E. (1962), On the theory of the social change, Homewood, Illinois, Dorset Press.

HANSEMARK O.C. (2003), "Need for achievement, locus of control and the prediction of business start-ups: A longitidunal study", Journal of Economic Psychology, 301-319.

KEEBLE D. et S. WALKER (1993), "New firms, small firms and dead firms : spatial patterns and determinants in the United Kingdom", Regional Studies, vol. ?, p.411-427. 
LACASSE R.M. et B. LAMBERT (1988), "La création d'une entreprise francophone minière", Revue Internationale de Gestion des Petites et Moyennes Organisations, vol. 3.

ROTTER J.B. (1971), "External control and internal control”, Psychology Today, 1971, 37-42, 5859.

ROTTER J.B. (1966), “General expectancies for internal versus external control of reinforcement. Psychology Monographs, 80, (Whole No. 609).

SHAPERO A. (1982), Social dimensions of entrepreneurship. In: Kent, C. et al (eds), The Encyclopedia of Entrepreneurship. Prentice-Hall, Englewood Cliffs. pp $72-90$.

SHAPERO A. (1975), "The displaced, uncomfortable entrepreneur", Psychology Today, 9 (6), 8388.

STOREY D.J. (1994), Understanding The Small Business Sector, Routledge, London.

THIREAU V. (1993), Les nouvelles dynamiques spatiales : à la redécouverte des territoires, Editions L'Harmattan.

VOLERY T. et I. SERVAIS (2001), "Rapport 2000 sur l'entrepreneuriat en France", Global Entrepreneurship Monitor (G.E.M.), Ecole de Management, Lyon. 